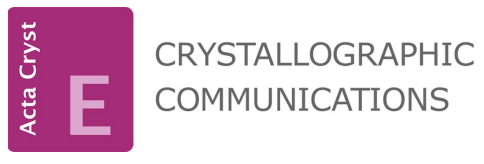

ISSN 2056-9890

Received 13 October 2015

Accepted 21 October 2015

Edited by $\mathrm{H}$. Stoeckli-Evans, University of Neuchâtel, Switzerland

Keywords: crystal structure; morpholine salts; phenoxyacetic acids; herbicides; 2,4-D; 3,5-D; hydrogen bonding

CCDC references: 1432389; 1432388; $1432387 ; 1432386$

Supporting information: this article has supporting information at journals.iucr.org/e

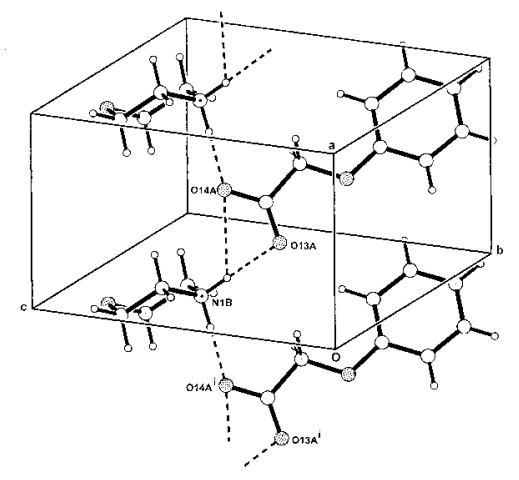

OPEN $\odot$ ACCESS

\section{Crystal structures and hydrogen bonding in the morpholinium salts of four phenoxyacetic acid analogues}

\author{
Graham Smith $^{\mathrm{a} *}$ and Daniel E. Lynch ${ }^{\mathrm{b}}$
}

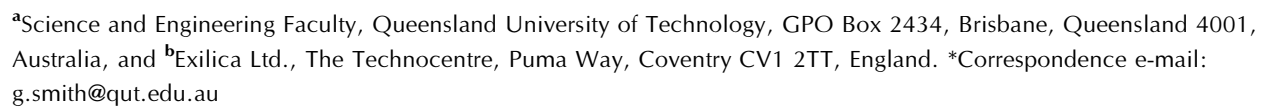

The anhydrous salts morpholinium (tetrahydro-2- $H$-1,4-oxazin-4-ium) phenoxyacetate, $\mathrm{C}_{4} \mathrm{H}_{10} \mathrm{NO}^{+} \cdot \mathrm{C}_{8} \mathrm{H}_{7} \mathrm{O}_{3}{ }^{-}$, (I), morpholinium (4-fluorophenoxy)acetate, $\mathrm{C}_{4} \mathrm{H}_{10} \mathrm{NO}^{+} \cdot \mathrm{C}_{8} \mathrm{H}_{6} \mathrm{FO}_{3}{ }^{-}$, (II), and isomeric morpholinium (3,5-dichlorophenoxy)acetate (3,5-D), (III), and morpholinium (2,4-dichlorophenoxy)acetic acid (2,4-D), $\mathrm{C}_{4} \mathrm{H}_{10} \mathrm{NO}^{+} \cdot \mathrm{C}_{8} \mathrm{H}_{5} \mathrm{Cl}_{2} \mathrm{O}_{3}{ }^{-}$, (IV), have been determined and their hydrogen-bonded structures are described. In the crystals of (I), (III) and (IV), one of the the aminium $\mathrm{H}$ atoms is involved in a three-centre asymmetric cation-anion $\mathrm{N}-\mathrm{H} \cdots \mathrm{O}, \mathrm{O}^{\prime} R_{1}^{2}(4)$ hydrogen-bonding interaction with the two carboxyl O-atom acceptors of the anion. With the structure of (II), the primary $\mathrm{N}-\mathrm{H} \cdots \mathrm{O}$ interaction is linear. In the structures of (I), (II) and (III), the second $\mathrm{N}-\mathrm{H} \cdots \mathrm{O}_{\text {carboxyl }}$ hydrogen bond generates one-dimensional chain structures extending in all cases along [100]. With (IV), the ion pairs are linked though inversion-related $\mathrm{N}-\mathrm{H} \cdots \mathrm{O}$ hydrogen bonds [graph set $R_{4}^{2}(8)$ ], giving a cyclic heterotetrameric structure.

\section{Chemical context}

Morpholine (tetrahydro-2- $H$-1,4-oxazine) is an moderately strong base $\left(\mathrm{p} K_{a}=8.33\right)$ and forms salts with a number of organic acids, some having medical applications, e.g. the salicylate (retarcyl, depasol), used as an analgesic, an antipyretic and an anti-inflammatory agent (O'Neil, 2001). The crystal structures of a number of these morpholinate compounds have been reported, some examples of salts with substituted benzoic acids being the 4-aminosalicylate (André et al., 2009), and a series of isomeric chloronitrobenzoates (2,4-, 2,5-, 4,2-, 4,3- and 5,2-) (Ishida et al., 2001a,b,c). In these, cation-anion hydrogen-bonding interactions generate either one-dimensional chains or discrete cyclic heterotetrameric structures. Of interest is the mode of hydrogen bonding in crystals of the morpholinium salts of some phenoxyacetic acid analogues, no

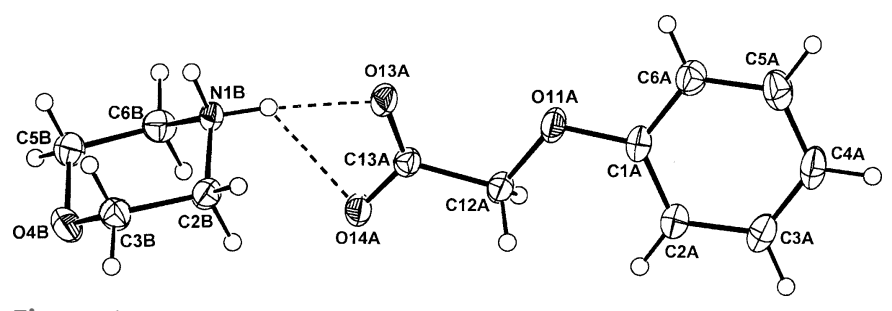

Figure 1

The atom-numbering scheme and the molecular conformation of the morpholinium cation $(B)$ and the phenoxyacetate anion $(A)$ in (I), with displacement ellipsoids drawn at the $40 \%$ probability level. The cationanion hydrogen bonds are shown as dashed lines. 
structures of which have been reported previously. The reaction of morpholine with phenoxyacetic acid (PAA), (4fluorophenoxy)acetic acid (PFPA) and with the two isomeric homologues, (3,5-dichlorophenoxy)acetic acid (3,5-D) and the herbicidally active (2,4-dichlorophenoxy)acetic acid (2,4-D) (Zumdahl, 2010), gave the anhydrous salts (I)-(IV), respectively. Their structures and hydrogen-bonding modes are reported on herein.
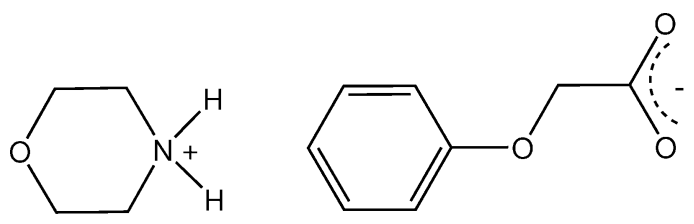

(I)
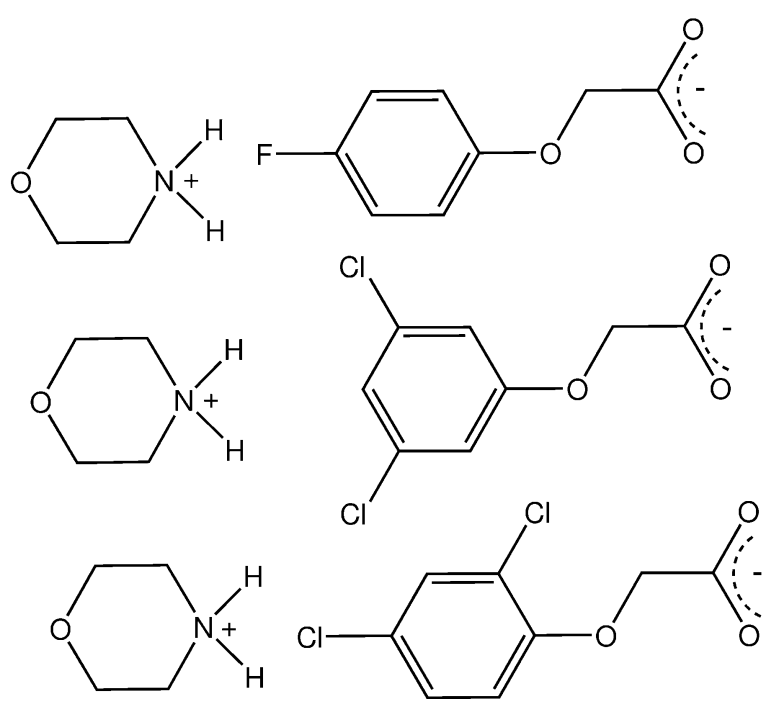

\section{Structural commentary}

The asymmetric units of (I)-(IV) comprise a morpholinium cation $(B)$ and a phenoxyacetate anion $(A)$ in (I) (Fig. 1), a (4fluorophenoxy)acetate anion $(A)$ in (II) (Fig. 2), a 3,5-dichlorophenoxyacetate anion (A) in (III) (Fig. 3) and a (2,4-

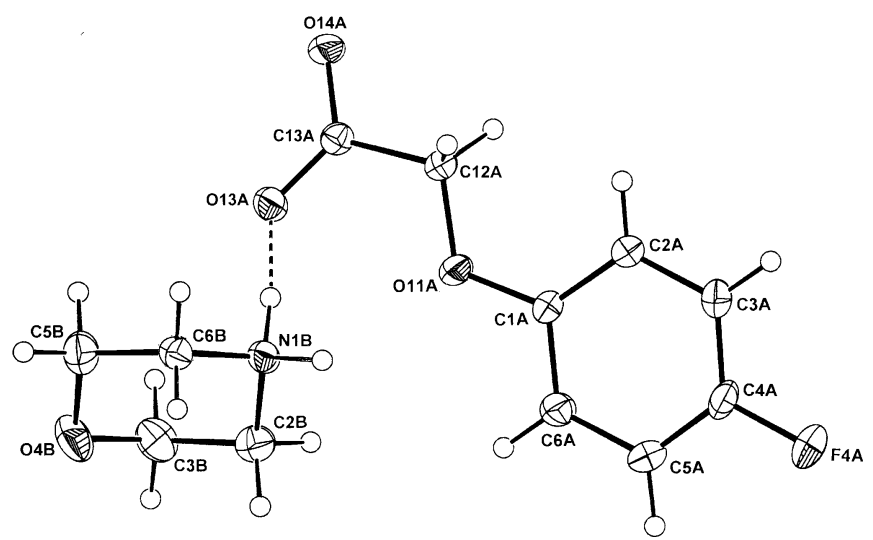

Figure 2

The atom-numbering scheme and the molecular conformation of the morpholinium cation $(B)$ and the 4-fluorophenoxy)acetate anion $(A)$ in (II), with displacement ellipsoids drawn at the $40 \%$ probability level. The cation-anion hydrogen bond is shown as a dashed line.

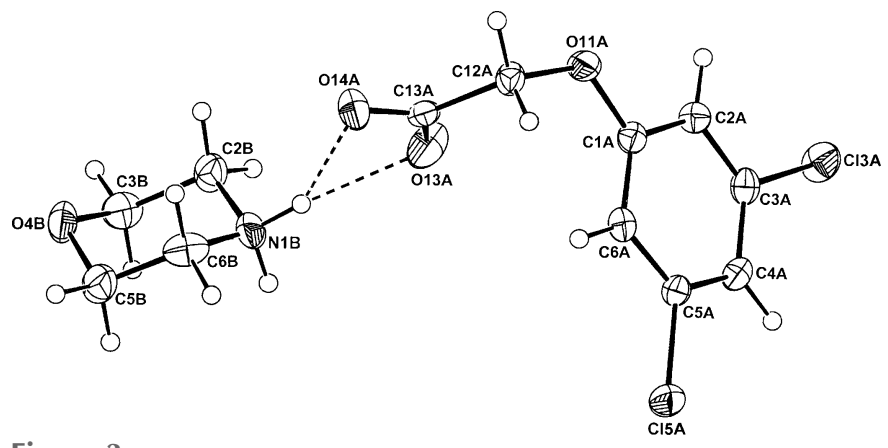

Figure 3

The atom-numbering scheme and the molecular conformation of the morpholinium cation $(B)$ and the $3,5-\mathrm{D}$ anion $(A)$ in (III), with displacement ellipsoids drawn at the $40 \%$ probability level. The cationanion hydrogen bonds are shown as dashed lines.

dichlorophenoxy)acetate anion (A) in (IV) (Fig. 4). The conformation of the oxoacetate side chains in the anions of (I) and (II) are essentially planar, with the defining torsion angle $\mathrm{C} 1 A-\mathrm{O} 11 A-\mathrm{C} 12 A-\mathrm{C} 13 A=176.75(14)$ and $176.53(14)^{\circ}$, respectively. This antiperiplanar $\left(180 \pm 30^{\circ}\right)$ conformation is similar to those of the parent acids PAA $\left(-175.1^{\circ}\right.$; Kennard et al., 1982), PFPA [176.0 (6) ${ }^{\circ}$; Smith et al., 1992] and their proton-transfer salts, e.g. the ammonium salts of PAA $\left[-177.48(18)^{\circ}\right]$ and PFPA $\left[-178.98(17)^{\circ}\right]$ (Smith, 2014). However, with the 3,5-D and 2,4-D salts, the side-chain conformations are both synclinal $\left(90 \pm 30^{\circ}\right)\left[-76.5(2)^{\circ}\right.$ in (III) and $72.91(19)^{\circ}$ in (IV)], similar to that in the parent acid 2,4-D (75.2 ; Smith et al., 1976), in the tryptaminium salt of 2,4-D [81.2 (6) $)^{\circ}$; Smith \& Lynch, 2015a] and in the 2:1 salt-adduct of 3,5-D with 4,4'-bipyridine [-71.6 (3) ${ }^{\circ}$; Lynch et al., 2003]. However, in the tryptaminium salt of 3,5-D (Smith \& Lynch, 2015b), the ammonium salts of both 2,4-D (Liu et al., 2009) and 3,5-D (Smith, 2014), the antiperiplanar conformation is

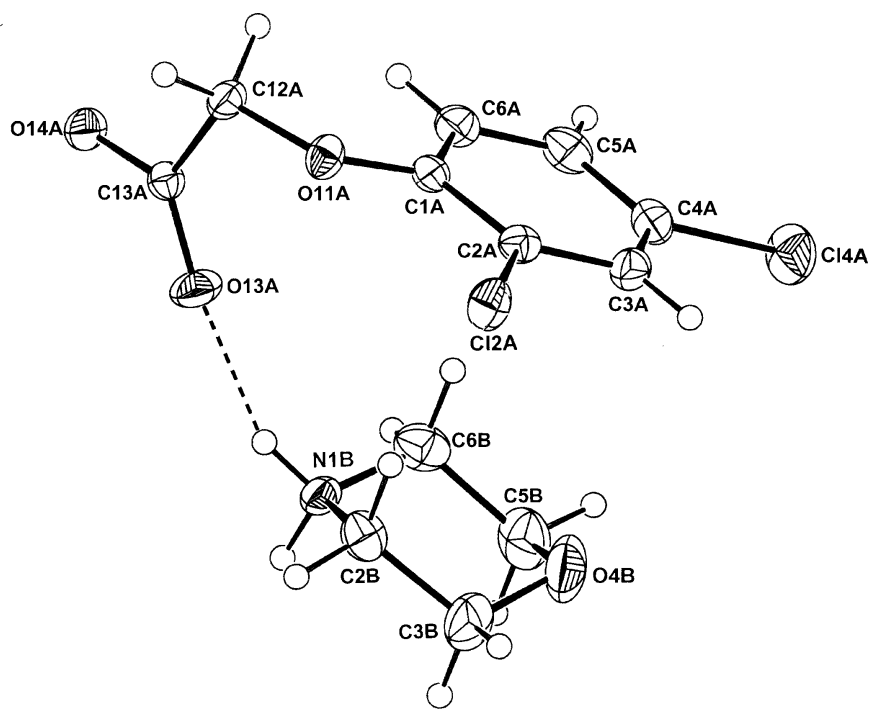

Figure 4

The atom-numbering scheme and the molecular conformation of the morpholinium cation $(B)$ and the 2,4-D anion $(A)$ in (IV), with displacement ellipsoids drawn at the $40 \%$ probability level. The cationanion hydrogen bonds are shown as dashed lines. 
Table 1

Hydrogen-bond geometry $\left(\AA{ }^{\circ}{ }^{\circ}\right)$ for (I).

\begin{tabular}{lllll}
\hline$D-\mathrm{H} \cdots A$ & $D-\mathrm{H}$ & $\mathrm{H} \cdots A$ & $D \cdots A$ & $D-\mathrm{H} \cdots A$ \\
\hline $\mathrm{N} 1 B-\mathrm{H} 11 B \cdots \mathrm{O} 13 A$ & $0.92(2)$ & $1.83(2)$ & $2.7366(18)$ & $169(2)$ \\
$\mathrm{N} 1 B-\mathrm{H} 11 B \cdots \mathrm{O} 14 A$ & $0.92(2)$ & $2.57(2)$ & $3.1655(17)$ & $123(1)$ \\
$\mathrm{N} 1 B-\mathrm{H} 12 B \cdots \mathrm{O} 14 A^{\mathrm{i}}$ & $0.95(1)$ & $1.76(1)$ & $2.7061(17)$ & $176(1)$ \\
$\mathrm{C} 4 A-\mathrm{H} 4 A \cdots \mathrm{O} 4 B^{\text {ii }}$ & 0.95 & 2.59 & $3.447(2)$ & 151 \\
$\mathrm{C} 6 B-\mathrm{H} 62 B \cdots \mathrm{O} 13 A^{\mathrm{iii}}$ & 0.99 & 2.39 & $3.148(2)$ & 133 \\
\hline
\end{tabular}

Symmetry codes: (i) $x-1, y, z$; (ii) $x+1, y+1, z-1$; (iii) $-x,-y+1,-z+1$.

Table 2

Hydrogen-bond geometry $\left(\AA,^{\circ}\right)$ for (II).

\begin{tabular}{lllll}
\hline$D-\mathrm{H} \cdots A$ & $D-\mathrm{H}$ & $\mathrm{H} \cdots A$ & $D \cdots A$ & $D-\mathrm{H} \cdots A$ \\
\hline $\mathrm{N} 1 B-\mathrm{H} 11 B \cdots \mathrm{O} 14 A^{\mathrm{i}}$ & $0.97(2)$ & $1.76(2)$ & $2.725(2)$ & $175(2)$ \\
$\mathrm{N} 1 B-\mathrm{H} 12 B \cdots \mathrm{O} 13 A$ & $0.94(2)$ & $1.80(2)$ & $2.718(2)$ & $165(2)$ \\
$\mathrm{C} 6 B-\mathrm{H} 61 B \cdots \mathrm{O} 14 A^{\mathrm{ii}}$ & 0.99 & 2.38 & $3.188(2)$ & 138 \\
\hline
\end{tabular}

Symmetry codes: (i) $x+1, y, z$; (ii) $-x,-y+2,-z+1$.

found [equivalent torsion angles $=-166.5(3), 172.61$ (8) and $-171.35(15)^{\circ}$, respectively].

\section{Supramolecular features}

In the crystals of both (I), (III) and (IV), a primary threecentre $R_{1}^{2}(4) \quad \mathrm{N} 1 B-\mathrm{H} \cdots\left(\mathrm{O}, \mathrm{O}^{\prime}\right)_{\text {carboxyl }}$ hydrogen-bonding interaction is present, with the asymmetry in (I) $[\mathrm{N} \cdots \mathrm{O}=$ 2.7366 (18) and 3.1655 (17) $\AA$ ] and (IV) [2.683 (2) and 3.115 (2) $\AA$ ] being significantly greater than that in (III) [2.892 (3) and 2.988 (3) ̊] (Tables 1, 3 and 4). With (II), the second $\mathrm{N}-\mathrm{H}$. . O d distance is 3.241 (2) $\AA$.

The hydrogen-bonding extensions involving the second aminium $\mathrm{H}$ atom of the cation result in different structures in

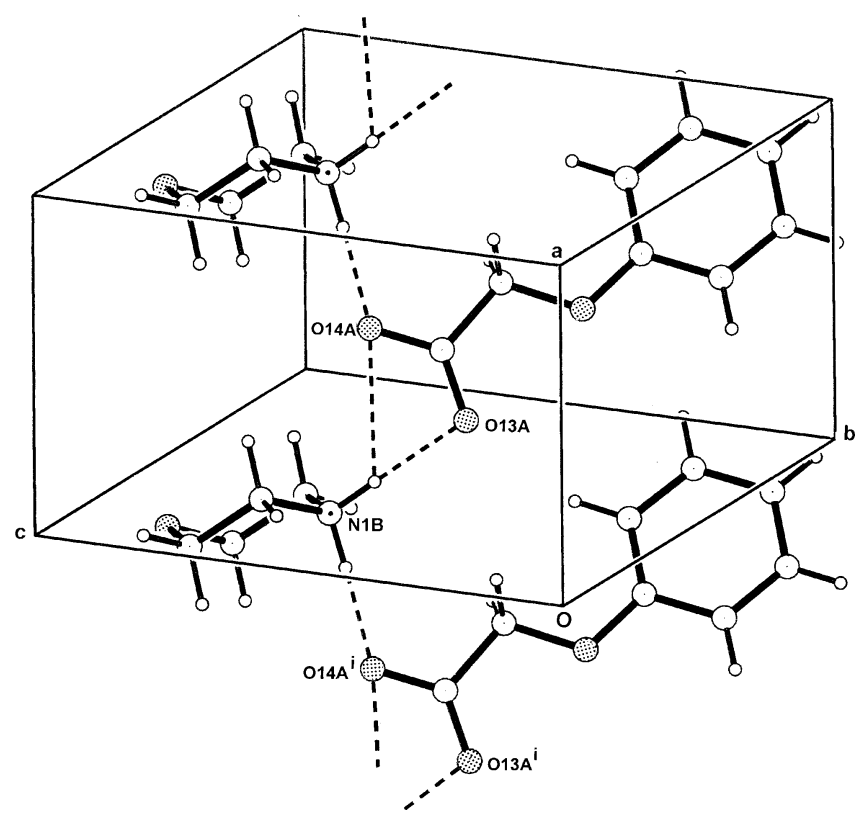

Figure 5

The one-dimensional hydrogen-bonded polymeric structure of (I) extending along $a$. For symmetry codes, see Table 1 .
Table 3

Hydrogen-bond geometry $\left(\AA,^{\circ}\right)$ for (III).

\begin{tabular}{lllll}
\hline$D-\mathrm{H} \cdots A$ & $D-\mathrm{H}$ & $\mathrm{H} \cdots A$ & $D \cdots A$ & $D-\mathrm{H} \cdots A$ \\
\hline $\mathrm{N} 1 B-\mathrm{H} 11 B \cdots \mathrm{O} 13 A$ & $0.88(2)$ & $2.07(2)$ & $2.892(3)$ & $156(2)$ \\
$\mathrm{N} 1 B-\mathrm{H} 11 B \cdots \mathrm{O} 14 A$ & $0.88(2)$ & $2.26(2)$ & $2.988(3)$ & $141(2)$ \\
$\mathrm{N} 1 B-\mathrm{H} 12 B \cdots \mathrm{O} 14 A^{\mathrm{i}}$ & $0.88(2)$ & $1.87(2)$ & $2.737(3)$ & $170(2)$ \\
$\mathrm{C} 12 A-\mathrm{H} 12 A \cdots \mathrm{O} 13 A^{\mathrm{ii}}$ & 0.99 & 2.41 & $3.398(3)$ & 173 \\
\hline
\end{tabular}

Symmetry codes: (i) $x-1, y, z$; (ii) $x+1, y, z$.

Table 4

Hydrogen-bond geometry $\left(\AA,^{\circ}\right)$ for (IV).

\begin{tabular}{|c|c|c|c|c|}
\hline$D-\mathrm{H} \cdots A$ & $D-\mathrm{H}$ & $\mathrm{H} \cdots A$ & $D \cdots A$ & $D-\mathrm{H} \cdots A$ \\
\hline $\mathrm{N} 1 B-\mathrm{H} 11 B \cdots \mathrm{O} 13 A^{\mathrm{i}}$ & $0.91(2)$ & $2.56(2)$ & $3.115(2)$ & $120(1)$ \\
\hline $\mathrm{N} 1 B-\mathrm{H} 11 B \cdots \mathrm{O} 14 A^{\mathrm{i}}$ & $0.91(2)$ & $1.79(2)$ & $2.683(2)$ & $169(2)$ \\
\hline $\mathrm{N} 1 B-\mathrm{H} 12 B \cdots \mathrm{O} 13 A$ & $0.87(2)$ & $1.92(2)$ & $2.747(2)$ & $158(2)$ \\
\hline $\mathrm{C} 12 A-\mathrm{H} 12 A \cdots \mathrm{O} 14 A^{\mathrm{ii}}$ & 0.99 & 2.50 & $3.484(2)$ & 173 \\
\hline $\mathrm{C} 2 B-\mathrm{H} 21 B \cdots \mathrm{O} 11 A^{\mathrm{iii}}$ & 0.99 & 2.57 & $3.477(2)$ & 151 \\
\hline $\mathrm{C} 5 B-\mathrm{H} 52 B \cdots \mathrm{O} 4 B^{\mathrm{iv}}$ & 0.99 & 2.58 & $3.489(3)$ & 153 \\
\hline
\end{tabular}

(I)-(III) compared to that in (IV). With (I)-(III), the primary heterodimers are all extended along $a$ through an N1B$\mathrm{H} \cdot \mathrm{O} 14 A^{\mathrm{i}}$ hydrogen bond (Tables $1-3$, respectively), into one-dimensional ribbon structures (Figs. 5-7). These ribbon structures provide further examples of the common hydrogen-

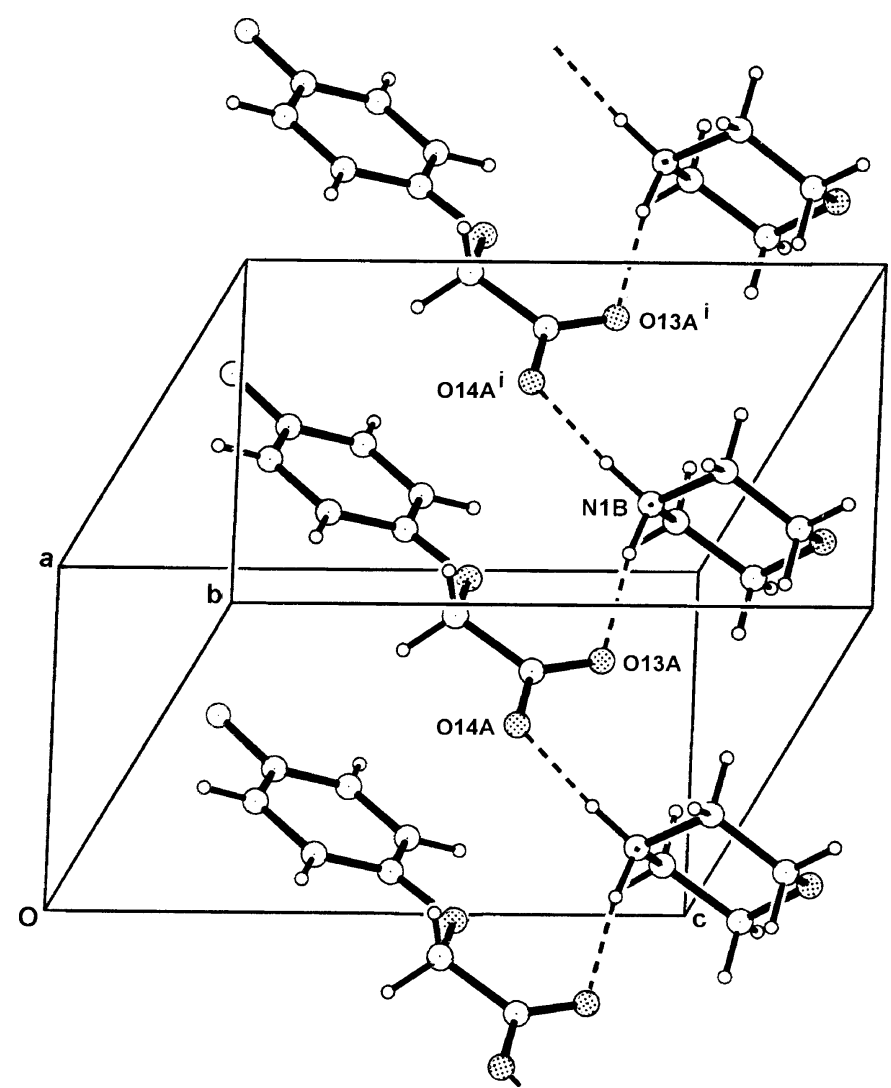

Figure 6

The one-dimensional hydrogen-bonded polymeric structure of (II) extending along $a$. For symmetry codes, see Table 2 . 


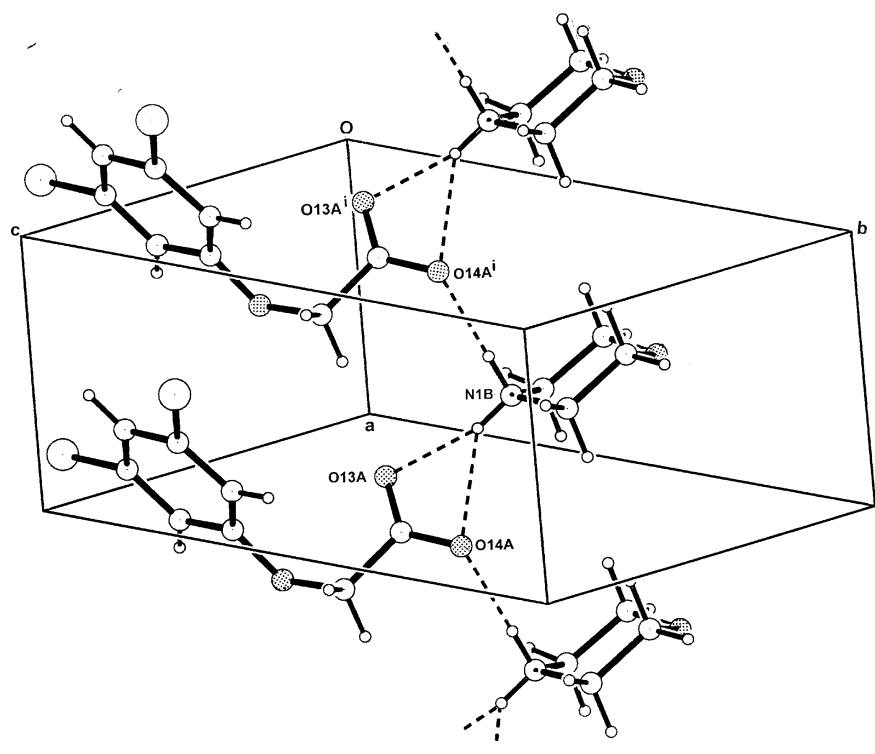

Figure 7

The one-dimensional hydrogen-bonded polymeric structure of (III) extending along $a$. For symmetry codes, see Table 3

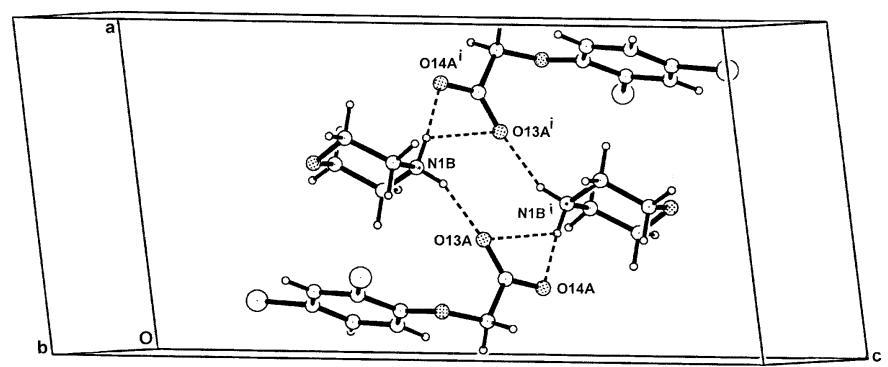

Figure 8

The cyclic hydrogen-bonded heterotetramer structure of (IV). For symmetry codes, see Table 4 .

bonded structure type found among the anhydrous aromatic morpholinium benzoate salts, e.g. with salicylic acid (Smith \& Lynch, 2015b) and with 2-chloro-4-nitrobenzoic acid (Ishida et $a l ., 2001 a$ ). In both of these examples, helical chains extend along $2_{1}$ screw axes in the crystals. Present also in structures of (I)-(IV) are minor weak inter-unit $\mathrm{C}-\mathrm{H} \cdots \mathrm{O}$ interactions: in (I), $\mathrm{C} 4 A-\mathrm{H} \cdots \mathrm{O} 4 B^{\mathrm{ii}}$ (Table 1); in (II), $\mathrm{C} 4 A-\mathrm{H} \cdots \mathrm{O} 4 B^{\mathrm{ii}}$;

Table 5

Experimental details.

(I)

\section{Crystal data}

Chemical formula

$M_{\mathrm{r}}$

Crystal system, space group

Temperature (K)

$a, b, c(\AA)$

$\alpha, \beta, \gamma\left({ }^{\circ}\right)$

$V\left(\AA^{3}\right)$

$Z$

Radiation type

$\mu\left(\mathrm{mm}^{-1}\right)$

Crystal size (mm)

Data collection

Diffractometer

Absorption correction

$T_{\min }, T_{\max }$

No. of measured, independent and observed reflections

$R_{\text {int }}$

$(\sin \theta / \lambda)_{\max }\left(\AA^{-1}\right)$

$\mathrm{C}_{4} \mathrm{H}_{10} \mathrm{NO}^{+} \cdot \mathrm{C}_{8} \mathrm{H}_{7} \mathrm{O}_{3}{ }^{-}$
239.27
Triclinic, $P \overline{1}$
200
$5.7079(5), 9.7735(9)$,
$\quad 11.3586(10)$
$78.277(7), 86.171(7)$,
$\quad 77.512(7)$
$605.58(10)$
2
Mo $K \alpha$
0.10
$0.50 \times 0.15 \times 0.04$

Oxford Diffraction Gemini-S

CCD detector

Multi-scan (CrysAlis PRO;

Agilent, 2014)

$0.860,0.980$

$4172,2370,1765[I>2 \sigma(I)]$

\subsection{3}

0.617

Refinement

$R\left[F^{2}>2 \sigma\left(F^{2}\right)\right], w R\left(F^{2}\right), S$

No. of reflections

No. of parameters

No. of restraints

$\mathrm{H}$-atom treatment

(II)

\section{$\mathrm{C}_{4} \mathrm{H}_{10} \mathrm{NO}^{+} \cdot \mathrm{C}_{8} \mathrm{H}_{6} \mathrm{FO}_{3}^{-}$}

257.26

Triclinic, $P \overline{1}$

200

5.7997 (5), 10.2605 (10),

$10.4836(11)$

88.388 (8), 82.792 (8),

$80.325(8)$

$610.11(10)$

2

Mo $K \alpha$

0.11

$0.50 \times 0.25 \times 0.05$

Oxford Diffraction Gemini-S CCD detector

Multi-scan (CrysAlis PRO;

Agilent, 2014)

$0.488,0.980$

4984, 2394, $1743[I>2 \sigma(I)]$

0.033

0.617

$0.048,0.113,1.02$
2370
154
0
$\mathrm{H}$ atoms treated by a mixture
$\quad$ of independent and
$\quad$ constrained refinement

$0.16,-0.17$
$0.046,0.116,1.04$

2394

169

2

$\mathrm{H}$ atoms treated by a mixture of independent and constrained refinement
$0.19,-0.20$
(III)

\begin{tabular}{|c|}
\hline $\mathrm{C}_{4} \mathrm{H}_{10} \mathrm{NO}^{+} \cdot \mathrm{C}_{8} \mathrm{H}_{5} \mathrm{Cl}_{2} \mathrm{O}_{3}^{-}$ \\
\hline Triclinic, $P \overline{1}$ \\
\hline 200 \\
\hline $\begin{array}{l}5.1733(4), 11.3751(10), \\
11.7808(10)\end{array}$ \\
\hline $\begin{array}{l}86.904 \text { (7), } 85.106(7) \\
77.936(7)\end{array}$ \\
\hline $675.01(10)$ \\
\hline 2 \\
\hline $\begin{array}{l}\text { Mo } K \alpha \\
0.49\end{array}$ \\
\hline $0.50 \times 0.13 \times 0.10$ \\
\hline
\end{tabular}

Oxford Diffraction Gemini-S CCD detector

Multi-scan (CrysAlis PRO;

Agilent, 2014)

$0.903,0.989$

5616, 2646, $2096[I>2 \sigma(I)]$

0.027

0.617

$0.039,0.091,1.03$

2646

178

2

$\mathrm{H}$ atoms treated by a mixture

of independent and

constrained refinement
(IV)

\author{
$\mathrm{C}_{4} \mathrm{H}_{10} \mathrm{NO}^{+} \cdot \mathrm{C}_{8} \mathrm{H}_{5} \mathrm{Cl}_{2} \mathrm{O}_{3}{ }^{-}$ \\ 308.15 \\ Monoclinic, $P 2_{1} / c$ \\ 200 \\ 9.3657 (5), 7.1702 (3), \\ $21.1340(11)$ \\ 90, $97.981(5), 90$ \\ 1405.48 (12) \\ 4 \\ Mo $K \alpha$ \\ 0.47 \\ $0.35 \times 0.35 \times 0.12$
}

Oxford Diffraction Gemini-S CCD detector

Multi-scan (CrysAlis PRO;

Agilent, 2014)

$0.933,0.980$

6400, 2754, $2273[I .2 \sigma(I)]$

0.026

0.617

$0.038,0.091,1.04$

2754

178

2

$\mathrm{H}$ atoms treated by a mixture of independent and constrained refinement

$0.28,-0.26$

$0.24,-0.26$

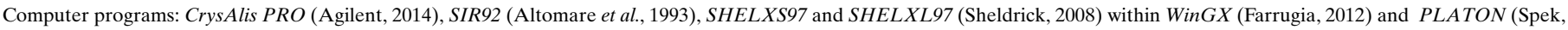
2009). 
$\mathrm{C} 6 B-\mathrm{H} \cdots \mathrm{O} 13 A^{\mathrm{iii}}$ (Table 2): in (III), $\mathrm{Cl} 2 A-\mathrm{H} \cdots \mathrm{O} 13 A^{\mathrm{ii}}$ (Table 3).

In the crystal of (IV), the second $\mathrm{N} 1 B-\mathrm{H} \cdots \mathrm{O} 14 A^{\mathrm{i}}$ hydrogen bond generates a centrosymmetric heterotetrameric ring structure [graph set $R_{4}^{2}(8)$ ] (Fig. 8). For symmetry code (i), see Table 4 . This cyclic system typifies the second structure type also found in a number of examples of morpholinium salts with ring-substituted benzoic acids, e.g. in the 2-chloro-5nitro-, 4-chloro-2-nitro-, 4-chloro-3-nitro- and 5-chloro-2nitrobenzoate series (Ishida et al., 2001a,b,c] and in the 4aminosalicylate (André et al., 2009).

Only weak inter-unit $\mathrm{C}-\mathrm{H} \cdots \mathrm{O}$ interactions to carboxyl or phenoxy O-atom acceptors are present in (IV) (Table 4), while no $\pi-\pi$ interactions are found in any of the structures.

\section{Synthesis and crystallization}

The title compounds (I)-(IV) were prepared by the dropwise addition of morpholine at room temperature to solutions of phenoxyacetic acid (150 mg), (4-fluorophenoxy)acetic (170 mg), (2,4-dichlorophenoxy)acetic acid or (2,4-dichlorophenoxy)acetic acid $(220 \mathrm{mg})$, respectively, in $15 \mathrm{ml}$ of ethanol. Room-temperature evaporation of the solutions gave either colourless plates of (III) or needles of (IV) from which specimens were cleaved for the X-ray analyses. For (I) and (II), the same preparative procedure was employed using phenoxyacetic acid or (4-fluorophenoxy)acetic acid but the final oils which resulted after solvent evaporation were redissolved in ethanol, finally giving thin colourless fragile plates of compounds (I) and (II) from which specimens were cleaved for the X-ray analyses.

\section{Refinement details}

Crystal data, data collection and structure refinement details are given in Table 5. $\mathrm{H}$ atoms were placed in calculated positions (aromatic $\mathrm{C}-\mathrm{H}=0.95 \AA$ or methylene $\mathrm{C}-\mathrm{H}=$ $0.99 \AA$ ) and were allowed to ride in the refinements, with $U_{\text {iso }}(\mathrm{H})=1.2 U_{\text {eq }}(\mathrm{C})$. The aminium $\mathrm{H}$ atoms were located in difference Fourier analyses and were allowed to refine with distance restraints $[\mathrm{N}-\mathrm{H}=0.90(2) \AA]$ and $U_{\text {iso }}(\mathrm{H})=$ $1.2 U_{\text {eq }}(\mathrm{N})$.

\section{Acknowledgements}

GS acknowledges financial support from the Science and Engineering Faculty, Queensland University of Technology.

\section{References}

Agilent (2014). CrysAlis PRO. Agilent Technologies Ltd, Yarnton, Oxfordshire, England.

Altomare, A., Cascarano, G., Giacovazzo, C. \& Guagliardi, A. (1993). J. Appl. Cryst. 26, 343-350.

André, V., Braga, D., Grepioni, F. \& Duarte, M. T. (2009). Cryst. Growth Des. 9, 5108-5116.

Farrugia, L. J. (2012). J. Appl. Cryst. 45, 849-854.

Ishida, H., Rahman, B. \& Kashino, S. (2001a). Acta Cryst. C57, 14501453.

Ishida, H., Rahman, B. \& Kashino, S. (2001b). Acta Cryst. E57, o627o629.

Ishida, H., Rahman, B. \& Kashino, S. (2001c). Acta Cryst. E57, o630o632.

Kennard, C. H. L., Smith, G. \& White, A. H. (1982). Acta Cryst. B38, 868-875.

Liu, H.-L., Guo, S.-H., Li, Y.-Y. \& Jian, F.-F. (2009). Acta Cryst. E65, o1905.

Lynch, D. E., Barfield, J., Frost, J., Antrobus, R. \& Simmons, J. (2003). Cryst. Eng. 6, 109-122.

O'Neil, M. J. (2001). Editor. The Merck Index, 13th ed., pp. 14951496. Whitehouse Station, NJ, USA: Merck \& Co. Inc.

Sheldrick, G. M. (2008). Acta Cryst. A64, 112-122.

Smith, G. (2014). Acta Cryst. E70, 528-532.

Smith, G., Kennard, C. H. L. \& White, A. H. (1976). J. Chem. Soc. Perkin Trans. 2, pp. 791-792.

Smith, G. \& Lynch, D. E. (2015a). Acta Cryst. E71, 671-674.

Smith, G. \& Lynch, D. E. (2015b). Unpublished results.

Smith, G., Lynch, D. E., Sagatys, D. S., Kennard, C. H. L. \& Katekar, G. F. (1992). Aust. J. Chem. 45, 1101-1108.

Spek, A. L. (2009). Acta Cryst. D65, 148-155.

Zumdahl, R. L. (2010). In A History of Weed Science in the United States. New York: Elsevier. 


\section{supporting information}

Acta Cryst. (2015). E71, 1392-1396 [https://doi.org/10.1107/S2056989015019842]

\section{Crystal structures and hydrogen bonding in the morpholinium salts of four} phenoxyacetic acid analogues

\section{Graham Smith and Daniel E. Lynch}

\section{Computing details}

For all compounds, data collection: CrysAlis PRO (Agilent, 2014); cell refinement: CrysAlis PRO (Agilent, 2014); data reduction: CrysAlis PRO (Agilent, 2014). Program(s) used to solve structure: SHELXS97 (Sheldrick, 2008) for (I), (II); SIR92 (Altomare et al., 1993) for (III), (IV). For all compounds, program(s) used to refine structure: SHELXL97 (Sheldrick, 2008) within WinGX (Farrugia, 2012); molecular graphics: PLATON (Spek, 2009); software used to prepare material for publication: PLATON (Spek, 2009).

(I) Tetrahydro-2H-1,4-oxazin-4-ium phenoxyacetate

Crystal data

$\mathrm{C}_{4} \mathrm{H}_{10} \mathrm{NO}^{+} \cdot \mathrm{C}_{8} \mathrm{H}_{7} \mathrm{O}_{3}^{-}$

$M_{r}=239.27$

Triclinic, $P \overline{1}$

Hall symbol: -P 1

$a=5.7079(5) \AA$

$b=9.7735(9) \AA$

$c=11.3586(10) \AA$

$\alpha=78.277(7)^{\circ}$

$\beta=86.171(7)^{\circ}$

$\gamma=77.512(7)^{\circ}$

$V=605.58(10) \AA^{3}$

Data collection

Oxford Diffraction Gemini-S CCD-detector diffractometer

Radiation source: Enhance (Mo) X-ray source

Graphite monochromator

Detector resolution: 16.077 pixels $\mathrm{mm}^{-1}$

$\omega$ scans

Absorption correction: multi-scan (CrysAlis PRO; Agilent, 2014)

$T_{\min }=0.860, T_{\max }=0.980$

\section{Refinement}

Refinement on $F^{2}$

Least-squares matrix: full

$R\left[F^{2}>2 \sigma\left(F^{2}\right)\right]=0.048$

$w R\left(F^{2}\right)=0.113$

$S=1.02$

2370 reflections
$Z=2$

$F(000)=256$

$D_{\mathrm{x}}=1.312 \mathrm{Mg} \mathrm{m}^{-3}$

Mo $K \alpha$ radiation, $\lambda=0.71073 \AA$

Cell parameters from 1029 reflections

$\theta=3.9-28.5^{\circ}$

$\mu=0.10 \mathrm{~mm}^{-1}$

$T=200 \mathrm{~K}$

Plate, colourless

$0.50 \times 0.15 \times 0.04 \mathrm{~mm}$

4172 measured reflections

2370 independent reflections

1765 reflections with $I>2 \sigma(I)$

$R_{\text {int }}=0.033$

$\theta_{\text {max }}=26.0^{\circ}, \theta_{\min }=3.1^{\circ}$

$h=-7 \rightarrow 6$

$k=-12 \rightarrow 12$

$l=-13 \rightarrow 13$

154 parameters

0 restraints

Primary atom site location: structure-invariant direct methods

Secondary atom site location: difference Fourier map 
Hydrogen site location: inferred from neighbouring sites

$\mathrm{H}$ atoms treated by a mixture of independent and constrained refinement

$$
\begin{gathered}
w=1 /\left[\sigma^{2}\left(F_{\mathrm{o}}^{2}\right)+(0.0456 P)^{2}\right] \\
\text { where } P=\left(F_{\mathrm{o}}^{2}+2 F_{\mathrm{c}}{ }^{2}\right) / 3 \\
(\Delta / \sigma)_{\max }<0.001 \\
\Delta \rho_{\max }=0.16 \mathrm{e} \AA^{-3} \\
\Delta \rho_{\min }=-0.17 \mathrm{e} \AA^{-3}
\end{gathered}
$$

Special details

Geometry. Bond distances, angles etc. have been calculated using the rounded fractional coordinates. All su's are estimated from the variances of the (full) variance-covariance matrix. The cell esds are taken into account in the estimation of distances, angles and torsion angles

Refinement. Refinement of $\mathrm{F}^{2}$ against ALL reflections. The weighted R-factor wR and goodness of fit $\mathrm{S}$ are based on $\mathrm{F}^{2}$,

\begin{tabular}{|c|c|c|c|c|}
\hline & $x$ & $y$ & $z$ & $U_{\text {iso }} * / U_{\text {eq }}$ \\
\hline O11A & $0.3926(2)$ & $0.81847(13)$ & $0.38178(10)$ & $0.0374(4)$ \\
\hline O13A & 0.11992 (19) & $0.65034(13)$ & $0.51716(10)$ & $0.0358(4)$ \\
\hline O14A & 0.41444 (19) & $0.54699(13)$ & $0.64451(10)$ & $0.0323(4)$ \\
\hline $\mathrm{C} 1 \mathrm{~A}$ & $0.5198(3)$ & $0.91643(18)$ & $0.32122(15)$ & $0.0303(5)$ \\
\hline $\mathrm{C} 2 \mathrm{~A}$ & $0.7157(3)$ & 0.94974 (19) & $0.36542(16)$ & $0.0346(6)$ \\
\hline C3A & $0.8267(3)$ & $1.0526(2)$ & $0.29642(17)$ & $0.0415(7)$ \\
\hline $\mathrm{C} 4 \mathrm{~A}$ & $0.7481(4)$ & $1.1208(2)$ & $0.18324(18)$ & $0.0449(7)$ \\
\hline C5A & $0.5549(4)$ & $1.0854(2)$ & $0.13875(17)$ & $0.0432(7)$ \\
\hline C6A & $0.4402(3)$ & $0.98450(19)$ & $0.20676(16)$ & $0.0365(6)$ \\
\hline $\mathrm{C} 12 \mathrm{~A}$ & 0.4895 & $0.73323(18)$ & $0.49189(14)$ & $0.0288(5)$ \\
\hline C13A & $0.3264(3)$ & $0.63578(18)$ & $0.55411(14)$ & $0.0268(5)$ \\
\hline O4B & $-0.1545(2)$ & $0.39037(15)$ & $0.94974(10)$ & $0.0445(5)$ \\
\hline N1B & $-0.1183(2)$ & $0.50356(15)$ & $0.70035(12)$ & $0.0270(4)$ \\
\hline $\mathrm{C} 2 \mathrm{~B}$ & $-0.1277(3)$ & $0.59359(19)$ & $0.79155(14)$ & $0.0322(6)$ \\
\hline C3B & -0.2632 & $0.5347(2)$ & $0.90159(15)$ & $0.0386(6)$ \\
\hline C5B & $-0.1515(4)$ & $0.3030(2)$ & $0.86300(16)$ & $0.0410(7)$ \\
\hline C6B & $-0.0136(3)$ & $0.35172(19)$ & $0.75076(15)$ & $0.0349(6)$ \\
\hline $\mathrm{H} 2 \mathrm{~A}$ & 0.77390 & 0.90240 & 0.44270 & $0.0420 *$ \\
\hline $\mathrm{H} 3 \mathrm{~A}$ & 0.95950 & 1.07660 & 0.32770 & $0.0500 *$ \\
\hline $\mathrm{H} 4 \mathrm{~A}$ & 0.82570 & 1.19120 & 0.13660 & $0.0540^{*}$ \\
\hline H5A & 0.50030 & 1.13090 & 0.06040 & $0.0520 *$ \\
\hline H6A & 0.30650 & 0.96140 & 0.17550 & $0.0440 *$ \\
\hline H11A & 0.51320 & 0.79650 & 0.54600 & $0.0350 *$ \\
\hline $\mathrm{H} 12 \mathrm{~A}$ & 0.64820 & 0.67470 & 0.47530 & $0.0350^{*}$ \\
\hline H11B & $-0.032(3)$ & $0.5424(18)$ & $0.6353(13)$ & $0.0320^{*}$ \\
\hline H12B & $-0.280(2)$ & 0.5164 (19) & $0.6782(13)$ & $0.0320^{*}$ \\
\hline $\mathrm{H} 21 \mathrm{~B}$ & -0.20870 & 0.69300 & 0.75840 & $0.0390^{*}$ \\
\hline $\mathrm{H} 22 \mathrm{~B}$ & 0.03720 & 0.59430 & 0.81310 & $0.0390^{*}$ \\
\hline H31B & -0.26710 & 0.59360 & 0.96330 & $0.0460 *$ \\
\hline H32B & -0.43070 & 0.53980 & 0.88040 & $0.0460 *$ \\
\hline H51B & -0.31840 & 0.30590 & 0.84220 & $0.0490 *$ \\
\hline
\end{tabular}
conventional R-factors $\mathrm{R}$ are based on $\mathrm{F}$, with $\mathrm{F}$ set to zero for negative $\mathrm{F}^{2}$. The threshold expression of $\mathrm{F}^{2}>2$ sigma $\left(\mathrm{F}^{2}\right)$ is used only for calculating R-factors ( $\mathrm{gt}$ ) etc. and is not relevant to the choice of reflections for refinement. R-factors based on $\mathrm{F}^{2}$ are statistically about twice as large as those based on F, and R- factors based on ALL data will be even larger.

Fractional atomic coordinates and isotropic or equivalent isotropic displacement parameters $\left(\AA^{2}\right)$ 
supporting information

\begin{tabular}{lllll} 
H52B & -0.07770 & 0.20290 & 0.89820 & $0.0490^{*}$ \\
H61B & 0.15700 & 0.34140 & 0.76960 & $0.0420^{*}$ \\
H62B & -0.02120 & 0.29200 & 0.69100 & $0.0420^{*}$ \\
\hline
\end{tabular}

Atomic displacement parameters $\left(\AA^{2}\right)$

\begin{tabular}{lllllll}
\hline & $U^{11}$ & $U^{22}$ & $U^{33}$ & $U^{12}$ & $U^{13}$ & $U^{23}$ \\
\hline O11A & $0.0348(7)$ & $0.0363(8)$ & $0.0397(7)$ & $-0.0186(6)$ & $-0.0046(5)$ & $0.0088(6)$ \\
O13A & $0.0252(7)$ & $0.0472(9)$ & $0.0342(7)$ & $-0.0162(6)$ & $-0.0014(5)$ & $0.0031(6)$ \\
O14A & $0.0270(6)$ & $0.0366(8)$ & $0.0316(7)$ & $-0.0121(6)$ & $-0.0004(5)$ & $0.0030(6)$ \\
C1A & $0.0299(9)$ & $0.0232(9)$ & $0.0364(10)$ & $-0.0081(8)$ & $0.0070(8)$ & $-0.0021(8)$ \\
C2A & $0.0351(10)$ & $0.0320(11)$ & $0.0373(10)$ & $-0.0128(8)$ & $0.0028(8)$ & $-0.0028(8)$ \\
C3A & $0.0387(11)$ & $0.0391(12)$ & $0.0509(12)$ & $-0.0201(9)$ & $0.0074(9)$ & $-0.0086(10)$ \\
C4A & $0.0515(12)$ & $0.0360(12)$ & $0.0487(12)$ & $-0.0222(10)$ & $0.0140(10)$ & $-0.0025(10)$ \\
C5A & $0.0545(12)$ & $0.0326(11)$ & $0.0387(11)$ & $-0.0117(10)$ & $0.0037(9)$ & $0.0030(9)$ \\
C6A & $0.0378(10)$ & $0.0307(11)$ & $0.0405(10)$ & $-0.0105(9)$ & $-0.0012(8)$ & $-0.0018(8)$ \\
C12A & $0.0255(9)$ & $0.0281(10)$ & $0.0328(9)$ & $-0.0101(8)$ & $0.0001(7)$ & $-0.0012(8)$ \\
C13A & $0.0257(9)$ & $0.0290(10)$ & $0.0273(9)$ & $-0.0093(8)$ & $0.0044(7)$ & $-0.0071(8)$ \\
O4B & $0.0641(9)$ & $0.0436(9)$ & $0.0267(7)$ & $-0.0196(7)$ & $-0.0014(6)$ & $0.0001(6)$ \\
N1B & $0.0239(7)$ & $0.0331(9)$ & $0.0247(7)$ & $-0.0120(7)$ & $0.0021(6)$ & $-0.0015(6)$ \\
C2B & $0.0333(10)$ & $0.0317(10)$ & $0.0342(10)$ & $-0.0119(8)$ & $0.0013(8)$ & $-0.0073(8)$ \\
C3B & $0.0458(11)$ & $0.0422(12)$ & $0.0295(10)$ & $-0.0137(9)$ & $0.0061(8)$ & $-0.0083(9)$ \\
C5B & $0.0577(13)$ & $0.0309(11)$ & $0.0363(10)$ & $-0.0180(9)$ & $-0.0055(9)$ & $-0.0003(8)$ \\
C6B & $0.0366(10)$ & $0.0313(11)$ & $0.0356(10)$ & $-0.0045(8)$ & $-0.0037(8)$ & $-0.0057(8)$ \\
& & & & & &
\end{tabular}

Geometric parameters $\left(\AA,{ }^{\circ}\right)$

\begin{tabular}{llll}
\hline $\mathrm{O} 11 \mathrm{~A}-\mathrm{C} 1 \mathrm{~A}$ & $1.372(2)$ & $\mathrm{C} 2 \mathrm{~A}-\mathrm{H} 2 \mathrm{~A}$ & 0.9500 \\
$\mathrm{O} 11 \mathrm{~A}-\mathrm{C} 12 \mathrm{~A}$ & $1.426(2)$ & $\mathrm{C} 3 \mathrm{~A}-\mathrm{H} 3 \mathrm{~A}$ & 0.9500 \\
$\mathrm{O} 13 \mathrm{~A}-\mathrm{C} 13 \mathrm{~A}$ & $1.247(2)$ & $\mathrm{C} 4 \mathrm{~A}-\mathrm{H} 4 \mathrm{~A}$ & 0.9500 \\
$\mathrm{O} 14 \mathrm{~A}-\mathrm{C} 13 \mathrm{~A}$ & $1.256(2)$ & $\mathrm{C} 5 \mathrm{~A}-\mathrm{H} 5 \mathrm{~A}$ & 0.9500 \\
$\mathrm{O} 4 \mathrm{~B}-\mathrm{C} 5 \mathrm{~B}$ & $1.426(2)$ & $\mathrm{C} 6 \mathrm{~A}-\mathrm{H} 6 \mathrm{~A}$ & 0.9500 \\
$\mathrm{O} 4 \mathrm{~B}-\mathrm{C} 3 \mathrm{~B}$ & $1.424(2)$ & $\mathrm{C} 12 \mathrm{~A}-\mathrm{H} 11 \mathrm{~A}$ & 0.9900 \\
$\mathrm{~N} 1 \mathrm{~B}-\mathrm{C} 6 \mathrm{~B}$ & $1.485(2)$ & $\mathrm{C} 12 \mathrm{~A}-\mathrm{H} 12 \mathrm{~A}$ & 0.9900 \\
$\mathrm{~N} 1 \mathrm{~B}-\mathrm{C} 2 \mathrm{~B}$ & $1.481(2)$ & $\mathrm{C} 2 \mathrm{~B}-\mathrm{C} 3 \mathrm{~B}$ & $1.504(2)$ \\
$\mathrm{N} 1 \mathrm{~B}-\mathrm{H} 12 \mathrm{~B}$ & $0.948(12)$ & $\mathrm{C} 5 \mathrm{~B}-\mathrm{C} 6 \mathrm{~B}$ & $1.501(3)$ \\
$\mathrm{N} 1 \mathrm{~B}-\mathrm{H} 11 \mathrm{~B}$ & $0.923(16)$ & $\mathrm{C} 2 \mathrm{~B}-\mathrm{H} 21 \mathrm{~B}$ & 0.9900 \\
$\mathrm{C} 1 \mathrm{~A}-\mathrm{C} 6 \mathrm{~A}$ & $1.391(2)$ & $\mathrm{C} 2 \mathrm{~B}-\mathrm{H} 22 \mathrm{~B}$ & 0.9900 \\
$\mathrm{C} 1 \mathrm{~A}-\mathrm{C} 2 \mathrm{~A}$ & $1.381(2)$ & $\mathrm{C} 3 \mathrm{~B}-\mathrm{H} 31 \mathrm{~B}$ & 0.9900 \\
$\mathrm{C} 2 \mathrm{~A}-\mathrm{C} 3 \mathrm{~A}$ & $1.384(3)$ & $\mathrm{C} 3 \mathrm{~B}-\mathrm{H} 32 \mathrm{~B}$ & 0.9900 \\
$\mathrm{C} 3 \mathrm{~A}-\mathrm{C} 4 \mathrm{~A}$ & $1.378(3)$ & $\mathrm{C} 5 \mathrm{~B}-\mathrm{H} 51 \mathrm{~B}$ & 0.9900 \\
$\mathrm{C} 4 \mathrm{~A}-\mathrm{C} 5 \mathrm{~A}$ & $1.379(3)$ & $\mathrm{C} 5 \mathrm{~B}-\mathrm{H} 52 \mathrm{~B}$ & 0.9900 \\
$\mathrm{C} 5 \mathrm{~A}-\mathrm{C} 6 \mathrm{~A}$ & $1.378(3)$ & $\mathrm{C} 6 \mathrm{~B}-\mathrm{H} 61 \mathrm{~B}$ & 0.9900 \\
$\mathrm{C} 12 \mathrm{~A}-\mathrm{C} 13 \mathrm{~A}$ & $1.515(2)$ & $\mathrm{C} 6 \mathrm{~B}-\mathrm{H} 62 \mathrm{~B}$ & 0.9900 \\
$\mathrm{C} 1 \mathrm{~A}-\mathrm{O} 11 \mathrm{~A}-\mathrm{C} 12 \mathrm{~A}$ & $116.65(13)$ & $\mathrm{C} 13 \mathrm{~A}-\mathrm{C} 12 \mathrm{~A}-\mathrm{H} 11 \mathrm{~A}$ & 109.00 \\
$\mathrm{C} 3 \mathrm{~B}-\mathrm{O} 4 \mathrm{~B}-\mathrm{C} 5 \mathrm{~B}$ & $110.28(13)$ & $\mathrm{O} 11 \mathrm{~A}-\mathrm{C} 12 \mathrm{~A}-\mathrm{H} 11 \mathrm{~A}$ & 109.00 \\
$\mathrm{C} 2 \mathrm{~B}-\mathrm{N} 1 \mathrm{~B}-\mathrm{C} 6 \mathrm{~B}$ & $110.91(13)$ & $\mathrm{C} 13 \mathrm{~A}-\mathrm{C} 12 \mathrm{~A}-\mathrm{H} 12 \mathrm{~A}$ & 109.00
\end{tabular}




$\begin{array}{ll}\text { C6B-N1B-H11B } & 113.4(11) \\ \text { C2B-N1B-H12B } & 104.8(10) \\ \text { H11B-N1B-H12B } & 109.0(14) \\ \text { C2B-N1B-H11B } & 106.3(10) \\ \text { C6B-N1B-H12B } & 111.9(11) \\ \text { O11A-C1A-C6A } & 115.56(15) \\ \text { C2A-C1A-C6A } & 119.48(16) \\ \text { O11A-C1A-C2A } & 124.96(15) \\ \text { C1A-C2A-C3A } & 119.58(16) \\ \text { C2A-C3A-C4A } & 121.20(18) \\ \text { C3A-C4A-C5A } & 118.97(19) \\ \text { C4A-C5A-C6A } & 120.65(18) \\ \text { C1A-C6A-C5A } & 120.10(17) \\ \text { O11A-C12A-C13A } & 111.82(14) \\ \text { O13A-C13A-O14A } & 125.13(16) \\ \text { O13A-C13A-C12A } & 120.24(14) \\ \text { O14A-C13A-C12A } & 114.59(15) \\ \text { C3A-C2A-H2A } & 120.00 \\ \text { C1A-C2A-H2A } & 120.00 \\ \text { C4A-C3A-H3A } & 119.00 \\ \text { C2A-C3A-H3A } & 119.00 \\ \text { C5A-C4A-H4A } & 121.00 \\ \text { C3A-C4A-H4A } & 121.00 \\ \text { C4A-C5A-H5A } & 120.00 \\ \text { C6A-C5A-H5A } & 120.00 \\ \text { C5A-C6A-H6A } & 120.00 \\ \text { C1A-C6A-H6A } & 120.00 \\ \text { C12A-O11A-C1A-C2A } & -8.1(2) \\ \text { C12A-O11A-C1A-C6A } & 171.64(15) \\ \text { C1A-O11A-C12A-C13A } & 176.53(14) \\ \text { C5B-O4B-C3B-C2B } & -60.23(18) \\ \text { C3B-O4B-C5B-C6B } & 59.8(2) \\ \text { C6B-N1B-C2B-C3B } & -55.34(17) \\ \text { C2B-N1B-C6B-C5B } & 54.74(18) \\ \text { C6A-C1A-C2A-C3A } & 1.5(3) \\ \text { O11A-C1A-C6A-C5A } & 179.61(16) \\ \text { C2A-C1A-C6A-C5A } & -0.7(3) \\ & \end{array}$

\begin{tabular}{|c|c|}
\hline $\mathrm{O} 11 \mathrm{~A}-\mathrm{C} 12 \mathrm{~A}-\mathrm{H} 12 \mathrm{~A}$ & 109.00 \\
\hline $\mathrm{H} 11 \mathrm{~A}-\mathrm{C} 12 \mathrm{~A}-\mathrm{H} 12 \mathrm{~A}$ & 108.00 \\
\hline $\mathrm{N} 1 \mathrm{~B}-\mathrm{C} 2 \mathrm{~B}-\mathrm{C} 3 \mathrm{~B}$ & $109.23(14)$ \\
\hline $\mathrm{O} 4 \mathrm{~B}-\mathrm{C} 3 \mathrm{~B}-\mathrm{C} 2 \mathrm{~B}$ & $111.23(14)$ \\
\hline $\mathrm{O} 4 \mathrm{~B}-\mathrm{C} 5 \mathrm{~B}-\mathrm{C} 6 \mathrm{~B}$ & $111.72(16)$ \\
\hline $\mathrm{N} 1 \mathrm{~B}-\mathrm{C} 6 \mathrm{~B}-\mathrm{C} 5 \mathrm{~B}$ & $109.35(14)$ \\
\hline $\mathrm{N} 1 \mathrm{~B}-\mathrm{C} 2 \mathrm{~B}-\mathrm{H} 21 \mathrm{~B}$ & 110.00 \\
\hline $\mathrm{N} 1 \mathrm{~B}-\mathrm{C} 2 \mathrm{~B}-\mathrm{H} 22 \mathrm{~B}$ & 110.00 \\
\hline $\mathrm{C} 3 \mathrm{~B}-\mathrm{C} 2 \mathrm{~B}-\mathrm{H} 21 \mathrm{~B}$ & 110.00 \\
\hline $\mathrm{C} 3 \mathrm{~B}-\mathrm{C} 2 \mathrm{~B}-\mathrm{H} 22 \mathrm{~B}$ & 110.00 \\
\hline $\mathrm{H} 21 \mathrm{~B}-\mathrm{C} 2 \mathrm{~B}-\mathrm{H} 22 \mathrm{~B}$ & 108.00 \\
\hline $\mathrm{O} 4 \mathrm{~B}-\mathrm{C} 3 \mathrm{~B}-\mathrm{H} 31 \mathrm{~B}$ & 109.00 \\
\hline $\mathrm{O} 4 \mathrm{~B}-\mathrm{C} 3 \mathrm{~B}-\mathrm{H} 32 \mathrm{~B}$ & 109.00 \\
\hline $\mathrm{C} 2 \mathrm{~B}-\mathrm{C} 3 \mathrm{~B}-\mathrm{H} 31 \mathrm{~B}$ & 109.00 \\
\hline $\mathrm{C} 2 \mathrm{~B}-\mathrm{C} 3 \mathrm{~B}-\mathrm{H} 32 \mathrm{~B}$ & 109.00 \\
\hline $\mathrm{H} 31 \mathrm{~B}-\mathrm{C} 3 \mathrm{~B}-\mathrm{H} 32 \mathrm{~B}$ & 108.00 \\
\hline $\mathrm{O} 4 \mathrm{~B}-\mathrm{C} 5 \mathrm{~B}-\mathrm{H} 51 \mathrm{~B}$ & 109.00 \\
\hline $\mathrm{O} 4 \mathrm{~B}-\mathrm{C} 5 \mathrm{~B}-\mathrm{H} 52 \mathrm{~B}$ & 109.00 \\
\hline $\mathrm{C} 6 \mathrm{~B}-\mathrm{C} 5 \mathrm{~B}-\mathrm{H} 51 \mathrm{~B}$ & 109.00 \\
\hline $\mathrm{C} 6 \mathrm{~B}-\mathrm{C} 5 \mathrm{~B}-\mathrm{H} 52 \mathrm{~B}$ & 109.00 \\
\hline $\mathrm{H} 51 \mathrm{~B}-\mathrm{C} 5 \mathrm{~B}-\mathrm{H} 52 \mathrm{~B}$ & 108.00 \\
\hline $\mathrm{N} 1 \mathrm{~B}-\mathrm{C} 6 \mathrm{~B}-\mathrm{H} 61 \mathrm{~B}$ & 110.00 \\
\hline $\mathrm{N} 1 \mathrm{~B}-\mathrm{C} 6 \mathrm{~B}-\mathrm{H} 62 \mathrm{~B}$ & 110.00 \\
\hline $\mathrm{C} 5 \mathrm{~B}-\mathrm{C} 6 \mathrm{~B}-\mathrm{H} 61 \mathrm{~B}$ & 110.00 \\
\hline $\mathrm{C} 5 \mathrm{~B}-\mathrm{C} 6 \mathrm{~B}-\mathrm{H} 62 \mathrm{~B}$ & 110.00 \\
\hline $\mathrm{H} 61 \mathrm{~B}-\mathrm{C} 6 \mathrm{~B}-\mathrm{H} 62 \mathrm{~B}$ & 108.00 \\
\hline $\mathrm{O} 11 \mathrm{~A}-\mathrm{C} 1 \mathrm{~A}-\mathrm{C} 2 \mathrm{~A}-\mathrm{C} 3 \mathrm{~A}$ & $-178.84(17)$ \\
\hline $\mathrm{C} 1 \mathrm{~A}-\mathrm{C} 2 \mathrm{~A}-\mathrm{C} 3 \mathrm{~A}-\mathrm{C} 4 \mathrm{~A}$ & $-1.2(3)$ \\
\hline $\mathrm{C} 2 \mathrm{~A}-\mathrm{C} 3 \mathrm{~A}-\mathrm{C} 4 \mathrm{~A}-\mathrm{C} 5 \mathrm{~A}$ & $0.1(3)$ \\
\hline $\mathrm{C} 3 \mathrm{~A}-\mathrm{C} 4 \mathrm{~A}-\mathrm{C} 5 \mathrm{~A}-\mathrm{C} 6 \mathrm{~A}$ & $0.7(3)$ \\
\hline $\mathrm{C} 4 \mathrm{~A}-\mathrm{C} 5 \mathrm{~A}-\mathrm{C} 6 \mathrm{~A}-\mathrm{C} 1 \mathrm{~A}$ & $-0.4(3)$ \\
\hline $\mathrm{O} 11 \mathrm{~A}-\mathrm{C} 12 \mathrm{~A}-\mathrm{C} 13 \mathrm{~A}-\mathrm{O} 14 \mathrm{~A}$ & $172.45(14)$ \\
\hline $\mathrm{O} 11 \mathrm{~A}-\mathrm{C} 12 \mathrm{~A}-\mathrm{C} 13 \mathrm{~A}-\mathrm{O} 13 \mathrm{~A}$ & $-9.8(2)$ \\
\hline $\mathrm{N} 1 \mathrm{~B}-\mathrm{C} 2 \mathrm{~B}-\mathrm{C} 3 \mathrm{~B}-\mathrm{O} 4 \mathrm{~B}$ & $58.00(18)$ \\
\hline $\mathrm{O} 4 \mathrm{~B}-\mathrm{C} 5 \mathrm{~B}-\mathrm{C} 6 \mathrm{~B}-\mathrm{N} 1 \mathrm{~B}$ & $-56.8(2)$ \\
\hline
\end{tabular}

Hydrogen-bond geometry $\left(\AA,{ }^{\circ}\right)$

\begin{tabular}{lllll}
\hline$D-\mathrm{H} \cdots A$ & $D-\mathrm{H}$ & $\mathrm{H} \cdots A$ & $D \cdots A$ & $D-\mathrm{H} \cdots A$ \\
\hline $\mathrm{N} 1 B-\mathrm{H} 11 B \cdots \mathrm{O} 13 A$ & $0.92(2)$ & $1.83(2)$ & $2.7366(18)$ & $169(2)$ \\
$\mathrm{N} 1 B-\mathrm{H} 11 B \cdots \mathrm{O} 14 A$ & $0.92(2)$ & $2.57(2)$ & $3.1655(17)$ & $123(1)$ \\
$\mathrm{N} 1 B-\mathrm{H} 12 B \cdots \mathrm{O} 14 A^{\mathrm{i}}$ & $0.95(1)$ & $1.76(1)$ & $2.7061(17)$ & $176(1)$
\end{tabular}




\begin{tabular}{lllll}
$\mathrm{C} 4 A-\mathrm{H} 4 A \cdots \mathrm{O} 4 B^{\mathrm{ii}}$ & 0.95 & 2.59 & $3.447(2)$ & 151 \\
$\mathrm{C} 6 B-\mathrm{H} 62 B \cdots \mathrm{O} 13 A^{\mathrm{iii}}$ & 0.99 & 2.39 & $3.148(2)$ & 133 \\
\hline
\end{tabular}

Symmetry codes: (i) $x-1, y, z$; (ii) $x+1, y+1, z-1$; (iii) $-x,-y+1,-z+1$.

(II) Tetrahydro-2H-1,4-oxazin-4-ium (4-fluorophenoxy)acetate

\section{Crystal data}

$$
\begin{aligned}
& \mathrm{C}_{4} \mathrm{H}_{10} \mathrm{NO}^{+} \cdot \mathrm{C}_{8} \mathrm{H}_{6} \mathrm{FO}_{3}{ }^{-} \\
& M_{r}=257.26 \\
& \text { Triclinic, } P \overline{1} \\
& \text { Hall symbol: -P 1 } \\
& a=5.7997(5) \AA \\
& b=10.2605(10) \AA \\
& c=10.4836(11) \AA \\
& \alpha=88.388(8)^{\circ} \\
& \beta=82.792(8)^{\circ} \\
& \gamma=80.325(8)^{\circ} \\
& V=610.11(10) \AA^{3}
\end{aligned}
$$

$$
\begin{aligned}
& Z=2 \\
& F(000)=272 \\
& D_{\mathrm{x}}=1.400 \mathrm{Mg} \mathrm{m}^{-3} \\
& \text { Mo } K \alpha \text { radiation, } \lambda=0.71073 \AA \\
& \text { Cell parameters from } 1163 \text { reflections } \\
& \theta=4.0-28.4^{\circ} \\
& \mu=0.11 \mathrm{~mm}^{-1} \\
& T=200 \mathrm{~K} \\
& \text { Plate, colourless } \\
& 0.50 \times 0.25 \times 0.05 \mathrm{~mm} \\
& \\
& 4984 \text { measured reflections } \\
& 2394 \text { independent reflections } \\
& 1743 \text { reflections with } I>2 \sigma(I) \\
& R_{\text {int }}=0.033 \\
& \theta_{\max }=26.0^{\circ}, \theta_{\min }=3.6^{\circ} \\
& h=-7 \rightarrow 6 \\
& k=-10 \rightarrow 12 \\
& l=-12 \rightarrow 12
\end{aligned}
$$

\section{Data collection}

Oxford Diffraction Gemini-S CCD-detector diffractometer

Radiation source: fine-focus sealed tube

Graphite monochromator

Detector resolution: 16.077 pixels $\mathrm{mm}^{-1}$

$\omega$ scans

Absorption correction: multi-scan

(CrysAlis PRO; Agilent, 2014)

$T_{\min }=0.488, T_{\max }=0.980$

\section{Refinement}

Refinement on $F^{2}$

Least-squares matrix: full

$R\left[F^{2}>2 \sigma\left(F^{2}\right)\right]=0.046$

$w R\left(F^{2}\right)=0.116$

$S=1.04$

2394 reflections

169 parameters

2 restraints

Primary atom site location: structure-invariant direct methods
51

133 
Fractional atomic coordinates and isotropic or equivalent isotropic displacement parameters $\left(\AA^{2}\right)$

\begin{tabular}{|c|c|c|c|c|}
\hline & $x$ & $y$ & $z$ & $U_{\text {iso }} * / U_{\text {eq }}$ \\
\hline F4A & 1.10905 (19) & $0.50899(12)$ & $0.11938(12)$ & $0.0518(4)$ \\
\hline O11A & $0.3317(2)$ & $0.71651(12)$ & $0.44716(12)$ & $0.0328(4)$ \\
\hline O13A & $-0.0039(2)$ & $0.82589(13)$ & $0.62958(12)$ & $0.0331(4)$ \\
\hline O14A & $-0.2493(2)$ & $0.88910(13)$ & $0.48447(12)$ & $0.0350(4)$ \\
\hline $\mathrm{C} 1 \mathrm{~A}$ & $0.5169(3)$ & $0.66778(16)$ & $0.35718(18)$ & $0.0255(6)$ \\
\hline $\mathrm{C} 2 \mathrm{~A}$ & 0.5357 & $0.69979(18)$ & $0.22788(18)$ & $0.0300(6)$ \\
\hline $\mathrm{C} 3 \mathrm{~A}$ & 0.7372 & 0.64655 (19) & 0.14698 (19) & $0.0357(6)$ \\
\hline $\mathrm{C} 4 \mathrm{~A}$ & $0.9096(3)$ & $0.56021(18)$ & $0.1981(2)$ & $0.0331(6)$ \\
\hline C5A & 0.8925 & $0.52405(18)$ & $0.3247(2)$ & $0.0333(6)$ \\
\hline C6A & 0.6948 & $0.57908(18)$ & $0.40512(19)$ & $0.0308(6)$ \\
\hline $\mathrm{C} 12 \mathrm{~A}$ & 0.1408 & $0.80714(17)$ & $0.40429(17)$ & $0.0263(6)$ \\
\hline C13A & $-0.0514(3)$ & $0.84287(16)$ & $0.51649(18)$ & $0.0249(6)$ \\
\hline O4B & $0.3300(2)$ & $0.84416(14)$ & $0.96211(13)$ & $0.0458(5)$ \\
\hline N1B & $0.4282(3)$ & $0.85297(15)$ & $0.68920(15)$ & $0.0288(5)$ \\
\hline $\mathrm{C} 2 \mathrm{~B}$ & 0.4898 & 0.72899 (19) & $0.76326(19)$ & $0.0357(7)$ \\
\hline $\mathrm{C} 3 \mathrm{~B}$ & $0.3244(4)$ & $0.7319(2)$ & $0.8863(2)$ & $0.0415(7)$ \\
\hline C5B & $0.2614(4)$ & $0.9620(2)$ & $0.8921(2)$ & $0.0425(7)$ \\
\hline C6B & $0.4214(3)$ & $0.96981(18)$ & $0.76981(19)$ & $0.0329(6)$ \\
\hline $\mathrm{H} 2 \mathrm{~A}$ & 0.41190 & 0.75780 & 0.19420 & $0.0360 *$ \\
\hline $\mathrm{H} 3 \mathrm{~A}$ & 0.75460 & 0.66960 & 0.05830 & $0.0430 *$ \\
\hline $\mathrm{H} 5 \mathrm{~A}$ & 1.01350 & 0.46260 & 0.35680 & $0.0400^{*}$ \\
\hline H6A & 0.68030 & 0.55610 & 0.49380 & $0.0370^{*}$ \\
\hline H11A & 0.07620 & 0.76630 & 0.33510 & $0.0320^{*}$ \\
\hline $\mathrm{H} 12 \mathrm{~A}$ & 0.19810 & 0.88810 & 0.36920 & $0.0320^{*}$ \\
\hline H11B & $0.541(3)$ & $0.861(2)$ & $0.6143(17)$ & $0.0500^{*}$ \\
\hline H12B & $0.283(3)$ & $0.855(2)$ & $0.6580(19)$ & $0.0500^{*}$ \\
\hline $\mathrm{H} 21 \mathrm{~B}$ & 0.47750 & 0.65180 & 0.71140 & $0.0430 *$ \\
\hline $\mathrm{H} 22 \mathrm{~B}$ & 0.65420 & 0.72040 & 0.78310 & $0.0430 *$ \\
\hline H31B & 0.36880 & 0.65030 & 0.93650 & $0.0500^{*}$ \\
\hline H32B & 0.16190 & 0.73360 & 0.86570 & $0.0500^{*}$ \\
\hline H51B & 0.26350 & 1.03940 & 0.94600 & $0.0510^{*}$ \\
\hline H52B & 0.09820 & 0.96510 & 0.87220 & $0.0510^{*}$ \\
\hline H61B & 0.36500 & 1.05120 & 0.72200 & $0.0390 *$ \\
\hline H62B & 0.58220 & 0.97440 & 0.78970 & $0.0390 *$ \\
\hline
\end{tabular}

Atomic displacement parameters $\left(\AA^{2}\right)$

\begin{tabular}{lllllll}
\hline & $U^{11}$ & $U^{22}$ & $U^{33}$ & $U^{12}$ & $U^{13}$ & $U^{23}$ \\
\hline F4A & $0.0376(7)$ & $0.0649(8)$ & $0.0429(8)$ & $0.0137(6)$ & $0.0067(6)$ & $-0.0140(6)$ \\
O11A & $0.0251(6)$ & $0.0409(8)$ & $0.0265(8)$ & $0.0079(5)$ & $0.0010(5)$ & $0.0031(6)$ \\
O13A & $0.0230(6)$ & $0.0513(8)$ & $0.0237(8)$ & $-0.0042(6)$ & $-0.0012(6)$ & $0.0015(6)$ \\
O14A & $0.0216(7)$ & $0.0497(8)$ & $0.0300(8)$ & $0.0041(6)$ & $-0.0036(6)$ & $0.0026(6)$ \\
C1A & $0.0231(9)$ & $0.0256(9)$ & $0.0259(11)$ & $-0.0003(7)$ & $-0.0003(8)$ & $-0.0031(8)$ \\
C2A & $0.0297(10)$ & $0.0304(10)$ & $0.0270(11)$ & $0.0040(8)$ & $-0.0045(8)$ & $-0.0024(8)$ \\
C3A & $0.0379(11)$ & $0.0405(11)$ & $0.0244(11)$ & $0.0025(9)$ & $0.0015(9)$ & $-0.0043(9)$
\end{tabular}




\begin{tabular}{lllllll} 
& & & & \\
C4A & $0.0265(10)$ & $0.0342(10)$ & $0.0346(13)$ & $0.0025(8)$ & $0.0039(8)$ & $-0.0122(9)$ \\
C5A & $0.0253(9)$ & $0.0337(10)$ & $0.0383(13)$ & $0.0040(8)$ & $-0.0053(9)$ & $-0.0006(9)$ \\
C6A & $0.0287(10)$ & $0.0344(10)$ & $0.0275(11)$ & $-0.0018(8)$ & $-0.0018(8)$ & $0.0043(9)$ \\
C12A & $0.0237(9)$ & $0.0273(9)$ & $0.0259(11)$ & $0.0011(7)$ & $-0.0022(8)$ & $0.0005(8)$ \\
C13A & $0.0224(9)$ & $0.0261(9)$ & $0.0266(11)$ & $-0.0053(7)$ & $-0.0023(8)$ & $-0.0003(8)$ \\
O4B & $0.0628(10)$ & $0.0521(9)$ & $0.0227(8)$ & $-0.0119(7)$ & $-0.0039(7)$ & $0.0049(7)$ \\
N1B & $0.0222(8)$ & $0.0415(9)$ & $0.0217(9)$ & $-0.0032(7)$ & $-0.0014(7)$ & $0.0008(7)$ \\
C2B & $0.0345(10)$ & $0.0336(11)$ & $0.0384(13)$ & $-0.0030(8)$ & $-0.0062(9)$ & $0.0006(9)$ \\
C3B & $0.0494(13)$ & $0.0433(12)$ & $0.0339(13)$ & $-0.0160(10)$ & $-0.0043(10)$ & $0.0084(10)$ \\
C5B & $0.0488(12)$ & $0.0450(13)$ & $0.0300(13)$ & $-0.0015(10)$ & $0.0015(10)$ & $-0.0025(10)$ \\
C6B & $0.0337(10)$ & $0.0346(11)$ & $0.0298(12)$ & $-0.0034(8)$ & $-0.0057(9)$ & $0.0034(9)$ \\
\hline
\end{tabular}

Geometric parameters $\left(\AA,{ }^{\circ}\right)$

\begin{tabular}{|c|c|c|c|}
\hline $\mathrm{F} 4 \mathrm{~A}-\mathrm{C} 4 \mathrm{~A}$ & $1.370(2)$ & $\mathrm{C} 12 \mathrm{~A}-\mathrm{C} 13 \mathrm{~A}$ & $1.523(3)$ \\
\hline $\mathrm{O} 11 \mathrm{~A}-\mathrm{C} 1 \mathrm{~A}$ & $1.373(2)$ & $\mathrm{C} 2 \mathrm{~A}-\mathrm{H} 2 \mathrm{~A}$ & 0.9500 \\
\hline $\mathrm{O} 11 \mathrm{~A}-\mathrm{C} 12 \mathrm{~A}$ & $1.431(2)$ & $\mathrm{C} 3 \mathrm{~A}-\mathrm{H} 3 \mathrm{~A}$ & 0.9500 \\
\hline $\mathrm{O} 13 \mathrm{~A}-\mathrm{C} 13 \mathrm{~A}$ & $1.251(2)$ & $\mathrm{C} 5 \mathrm{~A}-\mathrm{H} 5 \mathrm{~A}$ & 0.9500 \\
\hline $\mathrm{O} 14 \mathrm{~A}-\mathrm{C} 13 \mathrm{~A}$ & $1.249(2)$ & $\mathrm{C} 6 \mathrm{~A}-\mathrm{H} 6 \mathrm{~A}$ & 0.9500 \\
\hline $\mathrm{O} 4 \mathrm{~B}-\mathrm{C} 5 \mathrm{~B}$ & $1.422(2)$ & $\mathrm{C} 12 \mathrm{~A}-\mathrm{H} 11 \mathrm{~A}$ & 0.9900 \\
\hline $\mathrm{O} 4 \mathrm{~B}-\mathrm{C} 3 \mathrm{~B}$ & $1.425(2)$ & $\mathrm{C} 12 \mathrm{~A}-\mathrm{H} 12 \mathrm{~A}$ & 0.9900 \\
\hline $\mathrm{N} 1 \mathrm{~B}-\mathrm{C} 6 \mathrm{~B}$ & $1.478(2)$ & $\mathrm{C} 2 \mathrm{~B}-\mathrm{C} 3 \mathrm{~B}$ & $1.506(3)$ \\
\hline $\mathrm{N} 1 \mathrm{~B}-\mathrm{C} 2 \mathrm{~B}$ & $1.487(2)$ & $\mathrm{C} 5 \mathrm{~B}-\mathrm{C} 6 \mathrm{~B}$ & $1.494(3)$ \\
\hline N1B-H12B & $0.938(18)$ & $\mathrm{C} 2 \mathrm{~B}-\mathrm{H} 21 \mathrm{~B}$ & 0.9900 \\
\hline N1B-H11B & $0.968(18)$ & $\mathrm{C} 2 \mathrm{~B}-\mathrm{H} 22 \mathrm{~B}$ & 0.9900 \\
\hline $\mathrm{C} 1 \mathrm{~A}-\mathrm{C} 6 \mathrm{~A}$ & $1.391(3)$ & $\mathrm{C} 3 \mathrm{~B}-\mathrm{H} 31 \mathrm{~B}$ & 0.9900 \\
\hline $\mathrm{C} 1 \mathrm{~A}-\mathrm{C} 2 \mathrm{~A}$ & $1.381(3)$ & $\mathrm{C} 3 \mathrm{~B}-\mathrm{H} 32 \mathrm{~B}$ & 0.9900 \\
\hline $\mathrm{C} 2 \mathrm{~A}-\mathrm{C} 3 \mathrm{~A}$ & $1.395(3)$ & $\mathrm{C} 5 \mathrm{~B}-\mathrm{H} 51 \mathrm{~B}$ & 0.9900 \\
\hline $\mathrm{C} 3 \mathrm{~A}-\mathrm{C} 4 \mathrm{~A}$ & $1.372(3)$ & $\mathrm{C} 5 \mathrm{~B}-\mathrm{H} 52 \mathrm{~B}$ & 0.9900 \\
\hline $\mathrm{C} 4 \mathrm{~A}-\mathrm{C} 5 \mathrm{~A}$ & $1.364(3)$ & $\mathrm{C} 6 \mathrm{~B}-\mathrm{H} 61 \mathrm{~B}$ & 0.9900 \\
\hline $\mathrm{C} 5 \mathrm{~A}-\mathrm{C} 6 \mathrm{~A}$ & $1.382(3)$ & $\mathrm{C} 6 \mathrm{~B}-\mathrm{H} 62 \mathrm{~B}$ & 0.9900 \\
\hline $\mathrm{C} 1 \mathrm{~A}-\mathrm{O} 11 \mathrm{~A}-\mathrm{C} 12 \mathrm{~A}$ & $117.83(14)$ & $\mathrm{C} 13 \mathrm{~A}-\mathrm{C} 12 \mathrm{~A}-\mathrm{H} 11 \mathrm{~A}$ & 110.00 \\
\hline $\mathrm{C} 3 \mathrm{~B}-\mathrm{O} 4 \mathrm{~B}-\mathrm{C} 5 \mathrm{~B}$ & $109.82(15)$ & $\mathrm{O} 11 \mathrm{~A}-\mathrm{C} 12 \mathrm{~A}-\mathrm{H} 11 \mathrm{~A}$ & 110.00 \\
\hline $\mathrm{C} 2 \mathrm{~B}-\mathrm{N} 1 \mathrm{~B}-\mathrm{C} 6 \mathrm{~B}$ & $110.58(15)$ & $\mathrm{C} 13 \mathrm{~A}-\mathrm{C} 12 \mathrm{~A}-\mathrm{H} 12 \mathrm{~A}$ & 110.00 \\
\hline $\mathrm{C} 6 \mathrm{~B}-\mathrm{N} 1 \mathrm{~B}-\mathrm{H} 11 \mathrm{~B}$ & $106.7(12)$ & $\mathrm{O} 11 \mathrm{~A}-\mathrm{C} 12 \mathrm{~A}-\mathrm{H} 12 \mathrm{~A}$ & 110.00 \\
\hline $\mathrm{C} 2 \mathrm{~B}-\mathrm{N} 1 \mathrm{~B}-\mathrm{H} 12 \mathrm{~B}$ & $110.3(12)$ & $\mathrm{H} 11 \mathrm{~A}-\mathrm{C} 12 \mathrm{~A}-\mathrm{H} 12 \mathrm{~A}$ & 108.00 \\
\hline $\mathrm{H} 11 \mathrm{~B}-\mathrm{N} 1 \mathrm{~B}-\mathrm{H} 12 \mathrm{~B}$ & $105.8(16)$ & $\mathrm{N} 1 \mathrm{~B}-\mathrm{C} 2 \mathrm{~B}-\mathrm{C} 3 \mathrm{~B}$ & $109.48(16)$ \\
\hline $\mathrm{C} 2 \mathrm{~B}-\mathrm{N} 1 \mathrm{~B}-\mathrm{H} 11 \mathrm{~B}$ & $112.8(12)$ & $\mathrm{O} 4 \mathrm{~B}-\mathrm{C} 3 \mathrm{~B}-\mathrm{C} 2 \mathrm{~B}$ & $111.75(17)$ \\
\hline $\mathrm{C} 6 \mathrm{~B}-\mathrm{N} 1 \mathrm{~B}-\mathrm{H} 12 \mathrm{~B}$ & $110.5(12)$ & $\mathrm{O} 4 \mathrm{~B}-\mathrm{C} 5 \mathrm{~B}-\mathrm{C} 6 \mathrm{~B}$ & $111.70(17)$ \\
\hline $\mathrm{O} 11 \mathrm{~A}-\mathrm{C} 1 \mathrm{~A}-\mathrm{C} 6 \mathrm{~A}$ & $114.80(16)$ & $\mathrm{N} 1 \mathrm{~B}-\mathrm{C} 6 \mathrm{~B}-\mathrm{C} 5 \mathrm{~B}$ & $110.52(15)$ \\
\hline $\mathrm{C} 2 \mathrm{~A}-\mathrm{C} 1 \mathrm{~A}-\mathrm{C} 6 \mathrm{~A}$ & $119.88(17)$ & $\mathrm{N} 1 \mathrm{~B}-\mathrm{C} 2 \mathrm{~B}-\mathrm{H} 21 \mathrm{~B}$ & 110.00 \\
\hline $\mathrm{O} 11 \mathrm{~A}-\mathrm{C} 1 \mathrm{~A}-\mathrm{C} 2 \mathrm{~A}$ & $125.33(16)$ & $\mathrm{N} 1 \mathrm{~B}-\mathrm{C} 2 \mathrm{~B}-\mathrm{H} 22 \mathrm{~B}$ & 110.00 \\
\hline $\mathrm{C} 1 \mathrm{~A}-\mathrm{C} 2 \mathrm{~A}-\mathrm{C} 3 \mathrm{~A}$ & $119.71(17)$ & $\mathrm{C} 3 \mathrm{~B}-\mathrm{C} 2 \mathrm{~B}-\mathrm{H} 21 \mathrm{~B}$ & 110.00 \\
\hline $\mathrm{C} 2 \mathrm{~A}-\mathrm{C} 3 \mathrm{~A}-\mathrm{C} 4 \mathrm{~A}$ & $118.64(18)$ & $\mathrm{C} 3 \mathrm{~B}-\mathrm{C} 2 \mathrm{~B}-\mathrm{H} 22 \mathrm{~B}$ & 110.00 \\
\hline $\mathrm{C} 3 \mathrm{~A}-\mathrm{C} 4 \mathrm{~A}-\mathrm{C} 5 \mathrm{~A}$ & $122.83(18)$ & $\mathrm{H} 21 \mathrm{~B}-\mathrm{C} 2 \mathrm{~B}-\mathrm{H} 22 \mathrm{~B}$ & 108.00 \\
\hline $\mathrm{F} 4 \mathrm{~A}-\mathrm{C} 4 \mathrm{~A}-\mathrm{C} 5 \mathrm{~A}$ & $118.33(16)$ & $\mathrm{O} 4 \mathrm{~B}-\mathrm{C} 3 \mathrm{~B}-\mathrm{H} 31 \mathrm{~B}$ & 109.00 \\
\hline $\mathrm{F} 4 \mathrm{~A}-\mathrm{C} 4 \mathrm{~A}-\mathrm{C} 3 \mathrm{~A}$ & $118.84(18)$ & $\mathrm{O} 4 \mathrm{~B}-\mathrm{C} 3 \mathrm{~B}-\mathrm{H} 32 \mathrm{~B}$ & 109.00 \\
\hline
\end{tabular}




\begin{tabular}{|c|c|c|c|}
\hline $\mathrm{C} 4 \mathrm{~A}-\mathrm{C} 5 \mathrm{~A}-\mathrm{C} 6 \mathrm{~A}$ & $118.38(17)$ & $\mathrm{C} 2 \mathrm{~B}-\mathrm{C} 3 \mathrm{~B}-\mathrm{H} 31 \mathrm{~B}$ & 109.00 \\
\hline $\mathrm{C} 1 \mathrm{~A}-\mathrm{C} 6 \mathrm{~A}-\mathrm{C} 5 \mathrm{~A}$ & $120.53(18)$ & $\mathrm{C} 2 \mathrm{~B}-\mathrm{C} 3 \mathrm{~B}-\mathrm{H} 32 \mathrm{~B}$ & 109.00 \\
\hline $\mathrm{O} 11 \mathrm{~A}-\mathrm{C} 12 \mathrm{~A}-\mathrm{C} 13 \mathrm{~A}$ & $109.56(14)$ & $\mathrm{H} 31 \mathrm{~B}-\mathrm{C} 3 \mathrm{~B}-\mathrm{H} 32 \mathrm{~B}$ & 108.00 \\
\hline $\mathrm{O} 13 \mathrm{~A}-\mathrm{C} 13 \mathrm{~A}-\mathrm{O} 14 \mathrm{~A}$ & $125.43(17)$ & $\mathrm{O} 4 \mathrm{~B}-\mathrm{C} 5 \mathrm{~B}-\mathrm{H} 51 \mathrm{~B}$ & 109.00 \\
\hline $\mathrm{O} 14 \mathrm{~A}-\mathrm{C} 13 \mathrm{~A}-\mathrm{C} 12 \mathrm{~A}$ & $114.50(16)$ & $\mathrm{O} 4 \mathrm{~B}-\mathrm{C} 5 \mathrm{~B}-\mathrm{H} 52 \mathrm{~B}$ & 109.00 \\
\hline $\mathrm{O} 13 \mathrm{~A}-\mathrm{C} 13 \mathrm{~A}-\mathrm{C} 12 \mathrm{~A}$ & $120.06(15)$ & $\mathrm{C} 6 \mathrm{~B}-\mathrm{C} 5 \mathrm{~B}-\mathrm{H} 51 \mathrm{~B}$ & 109.00 \\
\hline $\mathrm{C} 3 \mathrm{~A}-\mathrm{C} 2 \mathrm{~A}-\mathrm{H} 2 \mathrm{~A}$ & 120.00 & $\mathrm{C} 6 \mathrm{~B}-\mathrm{C} 5 \mathrm{~B}-\mathrm{H} 52 \mathrm{~B}$ & 109.00 \\
\hline $\mathrm{C} 1 \mathrm{~A}-\mathrm{C} 2 \mathrm{~A}-\mathrm{H} 2 \mathrm{~A}$ & 120.00 & $\mathrm{H} 51 \mathrm{~B}-\mathrm{C} 5 \mathrm{~B}-\mathrm{H} 52 \mathrm{~B}$ & 108.00 \\
\hline $\mathrm{C} 4 \mathrm{~A}-\mathrm{C} 3 \mathrm{~A}-\mathrm{H} 3 \mathrm{~A}$ & 121.00 & $\mathrm{~N} 1 \mathrm{~B}-\mathrm{C} 6 \mathrm{~B}-\mathrm{H} 61 \mathrm{~B}$ & 110.00 \\
\hline $\mathrm{C} 2 \mathrm{~A}-\mathrm{C} 3 \mathrm{~A}-\mathrm{H} 3 \mathrm{~A}$ & 121.00 & $\mathrm{~N} 1 \mathrm{~B}-\mathrm{C} 6 \mathrm{~B}-\mathrm{H} 62 \mathrm{~B}$ & 110.00 \\
\hline $\mathrm{C} 4 \mathrm{~A}-\mathrm{C} 5 \mathrm{~A}-\mathrm{H} 5 \mathrm{~A}$ & 121.00 & $\mathrm{C} 5 \mathrm{~B}-\mathrm{C} 6 \mathrm{~B}-\mathrm{H} 61 \mathrm{~B}$ & 110.00 \\
\hline $\mathrm{C} 6 \mathrm{~A}-\mathrm{C} 5 \mathrm{~A}-\mathrm{H} 5 \mathrm{~A}$ & 121.00 & $\mathrm{C} 5 \mathrm{~B}-\mathrm{C} 6 \mathrm{~B}-\mathrm{H} 62 \mathrm{~B}$ & 110.00 \\
\hline $\mathrm{C} 5 \mathrm{~A}-\mathrm{C} 6 \mathrm{~A}-\mathrm{H} 6 \mathrm{~A}$ & 120.00 & $\mathrm{H} 61 \mathrm{~B}-\mathrm{C} 6 \mathrm{~B}-\mathrm{H} 62 \mathrm{~B}$ & 108.00 \\
\hline $\mathrm{C} 1 \mathrm{~A}-\mathrm{C} 6 \mathrm{~A}-\mathrm{H} 6 \mathrm{~A}$ & 120.00 & & \\
\hline $\mathrm{C} 12 \mathrm{~A}-\mathrm{O} 11 \mathrm{~A}-\mathrm{C} 1 \mathrm{~A}-\mathrm{C} 2 \mathrm{~A}$ & $0.5(2)$ & $\mathrm{C} 1 \mathrm{~A}-\mathrm{C} 2 \mathrm{~A}-\mathrm{C} 3 \mathrm{~A}-\mathrm{C} 4 \mathrm{~A}$ & $1.8(3)$ \\
\hline $\mathrm{C} 12 \mathrm{~A}-\mathrm{O} 11 \mathrm{~A}-\mathrm{C} 1 \mathrm{~A}-\mathrm{C} 6 \mathrm{~A}$ & $-179.26(15)$ & $\mathrm{C} 2 \mathrm{~A}-\mathrm{C} 3 \mathrm{~A}-\mathrm{C} 4 \mathrm{~A}-\mathrm{F} 4 \mathrm{~A}$ & $-179.19(16)$ \\
\hline $\mathrm{C} 1 \mathrm{~A}-\mathrm{O} 11 \mathrm{~A}-\mathrm{C} 12 \mathrm{~A}-\mathrm{C} 13 \mathrm{~A}$ & $176.75(14)$ & $\mathrm{C} 2 \mathrm{~A}-\mathrm{C} 3 \mathrm{~A}-\mathrm{C} 4 \mathrm{~A}-\mathrm{C} 5 \mathrm{~A}$ & $0.0(3)$ \\
\hline $\mathrm{C} 3 \mathrm{~B}-\mathrm{O} 4 \mathrm{~B}-\mathrm{C} 5 \mathrm{~B}-\mathrm{C} 6 \mathrm{~B}$ & $-59.8(2)$ & $\mathrm{C} 3 \mathrm{~A}-\mathrm{C} 4 \mathrm{~A}-\mathrm{C} 5 \mathrm{~A}-\mathrm{C} 6 \mathrm{~A}$ & $-1.4(3)$ \\
\hline $\mathrm{C} 5 \mathrm{~B}-\mathrm{O} 4 \mathrm{~B}-\mathrm{C} 3 \mathrm{~B}-\mathrm{C} 2 \mathrm{~B}$ & $60.3(2)$ & $\mathrm{F} 4 \mathrm{~A}-\mathrm{C} 4 \mathrm{~A}-\mathrm{C} 5 \mathrm{~A}-\mathrm{C} 6 \mathrm{~A}$ & $177.88(16)$ \\
\hline $\mathrm{C} 6 \mathrm{~B}-\mathrm{N} 1 \mathrm{~B}-\mathrm{C} 2 \mathrm{~B}-\mathrm{C} 3 \mathrm{~B}$ & $53.5(2)$ & $\mathrm{C} 4 \mathrm{~A}-\mathrm{C} 5 \mathrm{~A}-\mathrm{C} 6 \mathrm{~A}-\mathrm{C} 1 \mathrm{~A}$ & $0.8(3)$ \\
\hline $\mathrm{C} 2 \mathrm{~B}-\mathrm{N} 1 \mathrm{~B}-\mathrm{C} 6 \mathrm{~B}-\mathrm{C} 5 \mathrm{~B}$ & $-53.7(2)$ & $\mathrm{O} 11 \mathrm{~A}-\mathrm{C} 12 \mathrm{~A}-\mathrm{C} 13 \mathrm{~A}-\mathrm{O} 14 \mathrm{~A}$ & $-160.39(14)$ \\
\hline $\mathrm{C} 6 \mathrm{~A}-\mathrm{C} 1 \mathrm{~A}-\mathrm{C} 2 \mathrm{~A}-\mathrm{C} 3 \mathrm{~A}$ & $-2.3(3)$ & $\mathrm{O} 11 \mathrm{~A}-\mathrm{C} 12 \mathrm{~A}-\mathrm{C} 13 \mathrm{~A}-\mathrm{O} 13 \mathrm{~A}$ & $20.1(2)$ \\
\hline $\mathrm{O} 11 \mathrm{~A}-\mathrm{C} 1 \mathrm{~A}-\mathrm{C} 2 \mathrm{~A}-\mathrm{C} 3 \mathrm{~A}$ & $177.92(16)$ & $\mathrm{N} 1 \mathrm{~B}-\mathrm{C} 2 \mathrm{~B}-\mathrm{C} 3 \mathrm{~B}-\mathrm{O} 4 \mathrm{~B}$ & $-57.4(2)$ \\
\hline $\mathrm{C} 2 \mathrm{~A}-\mathrm{C} 1 \mathrm{~A}-\mathrm{C} 6 \mathrm{~A}-\mathrm{C} 5 \mathrm{~A}$ & $1.0(3)$ & $\mathrm{O} 4 \mathrm{~B}-\mathrm{C} 5 \mathrm{~B}-\mathrm{C} 6 \mathrm{~B}-\mathrm{N} 1 \mathrm{~B}$ & $57.1(2)$ \\
\hline $\mathrm{O} 11 \mathrm{~A}-\mathrm{C} 1 \mathrm{~A}-\mathrm{C} 6 \mathrm{~A}-\mathrm{C} 5 \mathrm{~A}$ & $-179.22(16)$ & & \\
\hline
\end{tabular}

Hydrogen-bond geometry $\left(\AA,{ }^{\circ}\right)$

\begin{tabular}{lllll}
\hline$D-\mathrm{H} \cdots A$ & $D-\mathrm{H}$ & $\mathrm{H} \cdots A$ & $D \cdots A$ & $D-\mathrm{H} \cdots A$ \\
\hline $\mathrm{N} 1 B-\mathrm{H} 11 B \cdots \mathrm{O} 14 A^{\mathrm{i}}$ & $0.97(2)$ & $1.76(2)$ & $2.725(2)$ & $175(2)$ \\
$\mathrm{N} 1 B-\mathrm{H} 12 B \cdots \mathrm{O} 13 A$ & $0.94(2)$ & $1.80(2)$ & $2.718(2)$ & $165(2)$ \\
$\mathrm{C} 6 B-\mathrm{H} 61 B \cdots \mathrm{O} 14 A^{\mathrm{ii}}$ & 0.99 & 2.38 & $3.188(2)$ & 138 \\
\hline
\end{tabular}

Symmetry codes: (i) $x+1, y, z$; (ii) $-x,-y+2,-z+1$.

(III) Tetrahydro-2H-1,4-oxazin-4-ium (3,5-dichlorophenoxy)acetate

Crystal data

$\mathrm{C}_{4} \mathrm{H}_{10} \mathrm{NO}^{+} \cdot \mathrm{C}_{8} \mathrm{H}_{5} \mathrm{Cl}_{2} \mathrm{O}_{3}^{-}$

$M_{r}=308.15$

Triclinic, $P \overline{1}$

Hall symbol: -P 1

$a=5.1733(4) \AA$

$b=11.3751(10) \AA$

$c=11.7808(10) \AA$

$\alpha=86.904(7)^{\circ}$

$\beta=85.106(7)^{\circ}$

$\gamma=77.936(7)^{\circ}$

$V=675.01(10) \AA^{3}$
$Z=2$

$F(000)=320$

$D_{\mathrm{x}}=1.516 \mathrm{Mg} \mathrm{m}^{-3}$

Mo $K \alpha$ radiation, $\lambda=0.71073 \AA$

Cell parameters from 1520 reflections

$\theta=3.7-27.8^{\circ}$

$\mu=0.49 \mathrm{~mm}^{-1}$

$T=200 \mathrm{~K}$

Needle, colourless

$0.50 \times 0.13 \times 0.10 \mathrm{~mm}$ 


\section{Data collection}

Oxford Diffraction Gemini-S CCD-detector diffractometer

Radiation source: fine-focus sealed tube Graphite monochromator

Detector resolution: 16.077 pixels $\mathrm{mm}^{-1}$ $\omega$ scans

Absorption correction: multi-scan

(CrysAlis PRO; Agilent, 2014)

$T_{\min }=0.903, T_{\max }=0.989$

Refinement

Refinement on $F^{2}$

Least-squares matrix: full

$R\left[F^{2}>2 \sigma\left(F^{2}\right)\right]=0.039$

$w R\left(F^{2}\right)=0.091$

$S=1.03$

2646 reflections

178 parameters

2 restraints

Primary atom site location: structure-invariant direct methods
5616 measured reflections

2646 independent reflections

2096 reflections with $I>2 \sigma(I)$

$R_{\text {int }}=0.027$

$\theta_{\max }=26.0^{\circ}, \theta_{\min }=3.5^{\circ}$

$h=-6 \rightarrow 6$

$k=-14 \rightarrow 12$

$l=-14 \rightarrow 14$
Secondary atom site location: difference Fourier map

Hydrogen site location: inferred from neighbouring sites

$\mathrm{H}$ atoms treated by a mixture of independent and constrained refinement

$w=1 /\left[\sigma^{2}\left(F_{\mathrm{o}}^{2}\right)+(0.0328 P)^{2}+0.273 P\right]$ where $P=\left(F_{\mathrm{o}}{ }^{2}+2 F_{\mathrm{c}}{ }^{2}\right) / 3$

$(\Delta / \sigma)_{\max }=0.001$

$\Delta \rho_{\max }=0.24 \mathrm{e} \AA^{-3}$

$\Delta \rho_{\min }=-0.26$ e $\AA^{-3}$

Special details

Geometry. Bond distances, angles etc. have been calculated using the rounded fractional coordinates. All su's are estimated from the variances of the (full) variance-covariance matrix. The cell esds are taken into account in the estimation of distances, angles and torsion angles

Refinement. Refinement of $\mathrm{F}^{2}$ against ALL reflections. The weighted R-factor $\mathrm{wR}$ and goodness of fit $\mathrm{S}$ are based on $\mathrm{F}^{2}$, conventional R-factors $R$ are based on $F$, with $F$ set to zero for negative $F^{2}$. The threshold expression of $F^{2}>2 \operatorname{sigma}\left(\mathrm{F}^{2}\right)$ is used only for calculating R-factors(gt) etc. and is not relevant to the choice of reflections for refinement. R-factors based on $\mathrm{F}^{2}$ are statistically about twice as large as those based on F, and R- factors based on ALL data will be even larger.

Fractional atomic coordinates and isotropic or equivalent isotropic displacement parameters $\left(\AA^{2}\right)$

\begin{tabular}{lllll}
\hline & $x$ & $y$ & $z$ & $U_{\text {iso }} * / U_{\text {eq }}$ \\
\hline C13A & $0.43721(11)$ & $0.33427(5)$ & $1.07395(5)$ & $0.0409(2)$ \\
C15A & $0.87160(12)$ & $-0.05932(5)$ & $0.83964(5)$ & $0.0423(2)$ \\
O11A & $1.2226(3)$ & $0.31888(13)$ & $0.77458(13)$ & $0.0356(5)$ \\
O13A & $0.8276(3)$ & $0.47790(19)$ & $0.67505(15)$ & $0.0575(7)$ \\
O14A & $1.0229(3)$ & $0.63014(15)$ & $0.69348(14)$ & $0.0476(6)$ \\
C1A & $1.0356(4)$ & $0.26801(19)$ & $0.83573(17)$ & $0.0271(6)$ \\
C2A & $0.8470(4)$ & $0.32703(19)$ & $0.91505(17)$ & $0.0281(6)$ \\
C3A & $0.6719(4)$ & $0.26282(19)$ & $0.97100(17)$ & $0.0279(6)$ \\
C4A & $0.6730(4)$ & $0.14542(19)$ & $0.94986(17)$ & $0.0307(7)$ \\
C5A & $0.8641(4)$ & $0.08971(19)$ & $0.87030(18)$ & $0.0303(7)$ \\
C6A & $1.0479(4)$ & $0.14815(19)$ & $0.81392(18)$ & $0.0300(7)$ \\
C12A & $1.2129(4)$ & $0.44437(19)$ & $0.77898(19)$ & $0.0306(7)$ \\
C13A & $1.0030(4)$ & $0.5221(2)$ & $0.70886(18)$ & $0.0335(7)$ \\
O4B & $0.3249(3)$ & $0.88990(15)$ & $0.45028(14)$ & $0.0502(6)$ \\
N1B & $0.4795(4)$ & $0.70096(19)$ & $0.61100(17)$ & $0.0416(7)$ \\
C2B & $0.5070(5)$ & $0.6814(2)$ & $0.48655(19)$ & $0.0395(8)$
\end{tabular}




\begin{tabular}{lllll} 
C3B & $0.3059(4)$ & $0.7719(2)$ & $0.4277(2)$ & $0.0418(8)$ \\
C5B & $0.4761(4)$ & $0.8273(2)$ & $0.6354(2)$ & $0.0415(8)$ \\
C6B & $0.2754(5)$ & $0.9090(2)$ & $0.5686(2)$ & $0.0466(8)$ \\
H2A & 0.83820 & 0.40890 & 0.93060 & $0.0340^{*}$ \\
H4A & 0.54740 & 0.10420 & 0.98840 & $0.0370^{*}$ \\
H6A & 1.18090 & 0.10720 & 0.76100 & $0.0360^{*}$ \\
H12A & 1.38860 & 0.46070 & 0.75130 & $0.0370^{*}$ \\
H13A & 1.17790 & 0.46790 & 0.85940 & $0.0370^{*}$ \\
H11B & $0.607(4)$ & $0.648(2)$ & $0.641(2)$ & $0.0560^{*}$ \\
H12B & $0.332(4)$ & $0.684(2)$ & $0.644(2)$ & $0.0560^{*}$ \\
H21B & 0.48270 & 0.59940 & 0.47290 & $0.0470^{*}$ \\
H22B & 0.68720 & 0.68820 & 0.45520 & $0.0470^{*}$ \\
H31B & 0.33260 & 0.76060 & 0.34450 & $0.0500^{*}$ \\
H32B & 0.12620 & 0.75950 & 0.45370 & $0.0500^{*}$ \\
H51B & 0.65360 & 0.84550 & 0.61490 & $0.0500^{*}$ \\
H52B & 0.43210 & 0.84010 & 0.71790 & $0.0500^{*}$ \\
H61B & 0.09660 & 0.89510 & 0.59410 & $0.0560^{*}$ \\
H62B & 0.27880 & 0.99360 & 0.58300 & $0.0560^{*}$ \\
\hline
\end{tabular}

Atomic displacement parameters $\left(\AA^{2}\right)$

\begin{tabular}{lllllll}
\hline & $U^{11}$ & $U^{22}$ & $U^{33}$ & $U^{12}$ & $U^{13}$ & $U^{23}$ \\
\hline C13A & $0.0453(3)$ & $0.0386(3)$ & $0.0341(3)$ & $-0.0032(3)$ & $0.0093(2)$ & $-0.0012(2)$ \\
C15A & $0.0563(4)$ & $0.0308(3)$ & $0.0431(3)$ & $-0.0179(3)$ & $0.0037(3)$ & $-0.0068(2)$ \\
O11A & $0.0296(8)$ & $0.0266(8)$ & $0.0495(10)$ & $-0.0083(6)$ & $0.0073(7)$ & $0.0017(7)$ \\
O13A & $0.0363(10)$ & $0.0903(15)$ & $0.0527(11)$ & $-0.0302(10)$ & $-0.0208(8)$ & $0.0323(10)$ \\
O14A & $0.0523(10)$ & $0.0345(10)$ & $0.0491(10)$ & $0.0042(8)$ & $-0.0036(8)$ & $0.0083(8)$ \\
C1A & $0.0251(10)$ & $0.0283(11)$ & $0.0291(11)$ & $-0.0076(9)$ & $-0.0058(9)$ & $0.0029(9)$ \\
C2A & $0.0310(11)$ & $0.0228(11)$ & $0.0310(11)$ & $-0.0054(9)$ & $-0.0070(9)$ & $0.0017(9)$ \\
C3A & $0.0291(11)$ & $0.0308(12)$ & $0.0226(10)$ & $-0.0040(9)$ & $-0.0026(8)$ & $0.0026(9)$ \\
C4A & $0.0329(12)$ & $0.0330(12)$ & $0.0274(11)$ & $-0.0113(9)$ & $-0.0018(9)$ & $0.0046(9)$ \\
C5A & $0.0375(12)$ & $0.0256(11)$ & $0.0296(11)$ & $-0.0085(9)$ & $-0.0083(9)$ & $0.0008(9)$ \\
C6A & $0.0296(11)$ & $0.0303(12)$ & $0.0292(11)$ & $-0.0048(9)$ & $-0.0011(9)$ & $-0.0009(9)$ \\
C12A & $0.0284(11)$ & $0.0287(12)$ & $0.0360(12)$ & $-0.0096(9)$ & $-0.0049(9)$ & $0.0064(9)$ \\
C13A & $0.0237(11)$ & $0.0456(15)$ & $0.0264(11)$ & $-0.0012(10)$ & $0.0042(9)$ & $0.0080(10)$ \\
O4B & $0.0674(12)$ & $0.0357(10)$ & $0.0378(10)$ & $0.0045(8)$ & $0.0049(8)$ & $0.0120(8)$ \\
N1B & $0.0414(12)$ & $0.0407(12)$ & $0.0338(11)$ & $0.0095(9)$ & $-0.0037(9)$ & $0.0096(9)$ \\
C2B & $0.0514(14)$ & $0.0278(13)$ & $0.0381(13)$ & $-0.0071(11)$ & $0.0001(11)$ & $-0.0015(10)$ \\
C3B & $0.0354(13)$ & $0.0572(17)$ & $0.0337(13)$ & $-0.0095(11)$ & $-0.0082(10)$ & $-0.0012(11)$ \\
C5B & $0.0343(13)$ & $0.0581(17)$ & $0.0367(13)$ & $-0.0181(11)$ & $0.0004(10)$ & $-0.0138(12)$ \\
C6B & $0.0626(16)$ & $0.0292(13)$ & $0.0433(14)$ & $-0.0040(12)$ & $0.0095(12)$ & $-0.0008(11)$ \\
& & & & & & \\
\hline
\end{tabular}

Geometric parameters $\left(\AA,{ }^{\circ}\right)$

\begin{tabular}{llll}
\hline $\mathrm{C} 13 \mathrm{~A}-\mathrm{C} 3 \mathrm{~A}$ & $1.746(2)$ & $\mathrm{C} 5 \mathrm{~A}-\mathrm{C} 6 \mathrm{~A}$ & $1.377(3)$ \\
$\mathrm{C} 15 \mathrm{~A}-\mathrm{C} 5 \mathrm{~A}$ & $1.744(2)$ & $\mathrm{C} 12 \mathrm{~A}-\mathrm{C} 13 \mathrm{~A}$ & $1.522(3)$ \\
$\mathrm{O} 11 \mathrm{~A}-\mathrm{C} 1 \mathrm{~A}$ & $1.364(3)$ & $\mathrm{C} 2 \mathrm{~A}-\mathrm{H} 2 \mathrm{~A}$ & 0.9500 \\
$\mathrm{O} 11 \mathrm{~A}-\mathrm{C} 12 \mathrm{~A}$ & $1.421(3)$ & $\mathrm{C} 4 \mathrm{~A}-\mathrm{H} 4 \mathrm{~A}$ & 0.9500
\end{tabular}




\begin{tabular}{|c|c|c|c|}
\hline $\mathrm{O} 13 \mathrm{~A}-\mathrm{C} 13 \mathrm{~A}$ & $1.228(3)$ & C6A-H6A & 0.9500 \\
\hline $\mathrm{O} 14 \mathrm{~A}-\mathrm{C} 13 \mathrm{~A}$ & $1.257(3)$ & $\mathrm{C} 12 \mathrm{~A}-\mathrm{H} 12 \mathrm{~A}$ & 0.9900 \\
\hline $\mathrm{O} 4 \mathrm{~B}-\mathrm{C} 6 \mathrm{~B}$ & $1.416(3)$ & $\mathrm{C} 12 \mathrm{~A}-\mathrm{H} 13 \mathrm{~A}$ & 0.9900 \\
\hline $\mathrm{O} 4 \mathrm{~B}-\mathrm{C} 3 \mathrm{~B}$ & $1.407(3)$ & $\mathrm{C} 2 \mathrm{~B}-\mathrm{C} 3 \mathrm{~B}$ & $1.490(3)$ \\
\hline $\mathrm{N} 1 \mathrm{~B}-\mathrm{C} 2 \mathrm{~B}$ & $1.485(3)$ & $\mathrm{C} 5 \mathrm{~B}-\mathrm{C} 6 \mathrm{~B}$ & $1.489(3)$ \\
\hline $\mathrm{N} 1 \mathrm{~B}-\mathrm{C} 5 \mathrm{~B}$ & $1.477(3)$ & $\mathrm{C} 2 \mathrm{~B}-\mathrm{H} 21 \mathrm{~B}$ & 0.9900 \\
\hline $\mathrm{N} 1 \mathrm{~B}-\mathrm{H} 12 \mathrm{~B}$ & $0.88(2)$ & $\mathrm{C} 2 \mathrm{~B}-\mathrm{H} 22 \mathrm{~B}$ & 0.9900 \\
\hline $\mathrm{N} 1 \mathrm{~B}-\mathrm{H} 11 \mathrm{~B}$ & $0.88(2)$ & $\mathrm{C} 3 \mathrm{~B}-\mathrm{H} 31 \mathrm{~B}$ & 0.9900 \\
\hline $\mathrm{C} 1 \mathrm{~A}-\mathrm{C} 2 \mathrm{~A}$ & $1.384(3)$ & $\mathrm{C} 3 \mathrm{~B}-\mathrm{H} 32 \mathrm{~B}$ & 0.9900 \\
\hline $\mathrm{C} 1 \mathrm{~A}-\mathrm{C} 6 \mathrm{~A}$ & $1.388(3)$ & $\mathrm{C} 5 \mathrm{~B}-\mathrm{H} 51 \mathrm{~B}$ & 0.9900 \\
\hline $\mathrm{C} 2 \mathrm{~A}-\mathrm{C} 3 \mathrm{~A}$ & $1.383(3)$ & $\mathrm{C} 5 \mathrm{~B}-\mathrm{H} 52 \mathrm{~B}$ & 0.9900 \\
\hline $\mathrm{C} 3 \mathrm{~A}-\mathrm{C} 4 \mathrm{~A}$ & $1.370(3)$ & $\mathrm{C} 6 \mathrm{~B}-\mathrm{H} 61 \mathrm{~B}$ & 0.9900 \\
\hline $\mathrm{C} 4 \mathrm{~A}-\mathrm{C} 5 \mathrm{~A}$ & $1.379(3)$ & $\mathrm{C} 6 \mathrm{~B}-\mathrm{H} 62 \mathrm{~B}$ & 0.9900 \\
\hline $\mathrm{C} 1 \mathrm{~A}-\mathrm{O} 11 \mathrm{~A}-\mathrm{C} 12 \mathrm{~A}$ & $120.24(17)$ & $\mathrm{C} 13 \mathrm{~A}-\mathrm{C} 12 \mathrm{~A}-\mathrm{H} 12 \mathrm{~A}$ & 109.00 \\
\hline $\mathrm{C} 3 \mathrm{~B}-\mathrm{O} 4 \mathrm{~B}-\mathrm{C} 6 \mathrm{~B}$ & $109.96(17)$ & $\mathrm{O} 11 \mathrm{~A}-\mathrm{C} 12 \mathrm{~A}-\mathrm{H} 12 \mathrm{~A}$ & 109.00 \\
\hline $\mathrm{C} 2 \mathrm{~B}-\mathrm{N} 1 \mathrm{~B}-\mathrm{C} 5 \mathrm{~B}$ & $111.72(18)$ & $\mathrm{C} 13 \mathrm{~A}-\mathrm{C} 12 \mathrm{~A}-\mathrm{H} 13 \mathrm{~A}$ & 109.00 \\
\hline $\mathrm{C} 5 \mathrm{~B}-\mathrm{N} 1 \mathrm{~B}-\mathrm{H} 11 \mathrm{~B}$ & $114.7(15)$ & $\mathrm{O} 11 \mathrm{~A}-\mathrm{C} 12 \mathrm{~A}-\mathrm{H} 13 \mathrm{~A}$ & 109.00 \\
\hline $\mathrm{C} 2 \mathrm{~B}-\mathrm{N} 1 \mathrm{~B}-\mathrm{H} 12 \mathrm{~B}$ & $112.3(15)$ & $\mathrm{H} 12 \mathrm{~A}-\mathrm{C} 12 \mathrm{~A}-\mathrm{H} 13 \mathrm{~A}$ & 108.00 \\
\hline $\mathrm{H} 11 \mathrm{~B}-\mathrm{N} 1 \mathrm{~B}-\mathrm{H} 12 \mathrm{~B}$ & $105(2)$ & $\mathrm{N} 1 \mathrm{~B}-\mathrm{C} 2 \mathrm{~B}-\mathrm{C} 3 \mathrm{~B}$ & 110.27 (19) \\
\hline $\mathrm{C} 2 \mathrm{~B}-\mathrm{N} 1 \mathrm{~B}-\mathrm{H} 11 \mathrm{~B}$ & $106.6(15)$ & $\mathrm{O} 4 \mathrm{~B}-\mathrm{C} 3 \mathrm{~B}-\mathrm{C} 2 \mathrm{~B}$ & $111.23(18)$ \\
\hline $\mathrm{C} 5 \mathrm{~B}-\mathrm{N} 1 \mathrm{~B}-\mathrm{H} 12 \mathrm{~B}$ & $106.7(15)$ & $\mathrm{N} 1 \mathrm{~B}-\mathrm{C} 5 \mathrm{~B}-\mathrm{C} 6 \mathrm{~B}$ & $109.70(19)$ \\
\hline $\mathrm{O} 11 \mathrm{~A}-\mathrm{C} 1 \mathrm{~A}-\mathrm{C} 6 \mathrm{~A}$ & $114.67(18)$ & $\mathrm{O} 4 \mathrm{~B}-\mathrm{C} 6 \mathrm{~B}-\mathrm{C} 5 \mathrm{~B}$ & $111.47(19)$ \\
\hline $\mathrm{C} 2 \mathrm{~A}-\mathrm{C} 1 \mathrm{~A}-\mathrm{C} 6 \mathrm{~A}$ & 120.75 (19) & $\mathrm{N} 1 \mathrm{~B}-\mathrm{C} 2 \mathrm{~B}-\mathrm{H} 21 \mathrm{~B}$ & 110.00 \\
\hline $\mathrm{O} 11 \mathrm{~A}-\mathrm{C} 1 \mathrm{~A}-\mathrm{C} 2 \mathrm{~A}$ & 124.57 (19) & $\mathrm{N} 1 \mathrm{~B}-\mathrm{C} 2 \mathrm{~B}-\mathrm{H} 22 \mathrm{~B}$ & 110.00 \\
\hline $\mathrm{C} 1 \mathrm{~A}-\mathrm{C} 2 \mathrm{~A}-\mathrm{C} 3 \mathrm{~A}$ & $117.77(19)$ & $\mathrm{C} 3 \mathrm{~B}-\mathrm{C} 2 \mathrm{~B}-\mathrm{H} 21 \mathrm{~B}$ & 110.00 \\
\hline $\mathrm{C} 13 \mathrm{~A}-\mathrm{C} 3 \mathrm{~A}-\mathrm{C} 4 \mathrm{~A}$ & $118.09(16)$ & $\mathrm{C} 3 \mathrm{~B}-\mathrm{C} 2 \mathrm{~B}-\mathrm{H} 22 \mathrm{~B}$ & 110.00 \\
\hline $\mathrm{C} 2 \mathrm{~A}-\mathrm{C} 3 \mathrm{~A}-\mathrm{C} 4 \mathrm{~A}$ & $123.29(19)$ & $\mathrm{H} 21 \mathrm{~B}-\mathrm{C} 2 \mathrm{~B}-\mathrm{H} 22 \mathrm{~B}$ & 108.00 \\
\hline $\mathrm{C} 13 \mathrm{~A}-\mathrm{C} 3 \mathrm{~A}-\mathrm{C} 2 \mathrm{~A}$ & $118.62(16)$ & $\mathrm{O} 4 \mathrm{~B}-\mathrm{C} 3 \mathrm{~B}-\mathrm{H} 31 \mathrm{~B}$ & 109.00 \\
\hline $\mathrm{C} 3 \mathrm{~A}-\mathrm{C} 4 \mathrm{~A}-\mathrm{C} 5 \mathrm{~A}$ & $117.18(19)$ & $\mathrm{O} 4 \mathrm{~B}-\mathrm{C} 3 \mathrm{~B}-\mathrm{H} 32 \mathrm{~B}$ & 109.00 \\
\hline $\mathrm{Cl} 5 \mathrm{~A}-\mathrm{C} 5 \mathrm{~A}-\mathrm{C} 6 \mathrm{~A}$ & $119.03(16)$ & $\mathrm{C} 2 \mathrm{~B}-\mathrm{C} 3 \mathrm{~B}-\mathrm{H} 31 \mathrm{~B}$ & 109.00 \\
\hline $\mathrm{C} 15 \mathrm{~A}-\mathrm{C} 5 \mathrm{~A}-\mathrm{C} 4 \mathrm{~A}$ & $118.85(16)$ & $\mathrm{C} 2 \mathrm{~B}-\mathrm{C} 3 \mathrm{~B}-\mathrm{H} 32 \mathrm{~B}$ & 109.00 \\
\hline $\mathrm{C} 4 \mathrm{~A}-\mathrm{C} 5 \mathrm{~A}-\mathrm{C} 6 \mathrm{~A}$ & $122.1(2)$ & $\mathrm{H} 31 \mathrm{~B}-\mathrm{C} 3 \mathrm{~B}-\mathrm{H} 32 \mathrm{~B}$ & 108.00 \\
\hline $\mathrm{C} 1 \mathrm{~A}-\mathrm{C} 6 \mathrm{~A}-\mathrm{C} 5 \mathrm{~A}$ & $118.84(19)$ & $\mathrm{N} 1 \mathrm{~B}-\mathrm{C} 5 \mathrm{~B}-\mathrm{H} 51 \mathrm{~B}$ & 110.00 \\
\hline $\mathrm{O} 11 \mathrm{~A}-\mathrm{C} 12 \mathrm{~A}-\mathrm{C} 13 \mathrm{~A}$ & $113.95(18)$ & $\mathrm{N} 1 \mathrm{~B}-\mathrm{C} 5 \mathrm{~B}-\mathrm{H} 52 \mathrm{~B}$ & 110.00 \\
\hline $\mathrm{O} 14 \mathrm{~A}-\mathrm{C} 13 \mathrm{~A}-\mathrm{C} 12 \mathrm{~A}$ & $115.08(19)$ & $\mathrm{C} 6 \mathrm{~B}-\mathrm{C} 5 \mathrm{~B}-\mathrm{H} 51 \mathrm{~B}$ & 110.00 \\
\hline $\mathrm{O} 13 \mathrm{~A}-\mathrm{C} 13 \mathrm{~A}-\mathrm{O} 14 \mathrm{~A}$ & $125.4(2)$ & $\mathrm{C} 6 \mathrm{~B}-\mathrm{C} 5 \mathrm{~B}-\mathrm{H} 52 \mathrm{~B}$ & 110.00 \\
\hline $\mathrm{O} 13 \mathrm{~A}-\mathrm{C} 13 \mathrm{~A}-\mathrm{C} 12 \mathrm{~A}$ & $119.5(2)$ & $\mathrm{H} 51 \mathrm{~B}-\mathrm{C} 5 \mathrm{~B}-\mathrm{H} 52 \mathrm{~B}$ & 108.00 \\
\hline $\mathrm{C} 3 \mathrm{~A}-\mathrm{C} 2 \mathrm{~A}-\mathrm{H} 2 \mathrm{~A}$ & 121.00 & $\mathrm{O} 4 \mathrm{~B}-\mathrm{C} 6 \mathrm{~B}-\mathrm{H} 61 \mathrm{~B}$ & 109.00 \\
\hline $\mathrm{C} 1 \mathrm{~A}-\mathrm{C} 2 \mathrm{~A}-\mathrm{H} 2 \mathrm{~A}$ & 121.00 & $\mathrm{O} 4 \mathrm{~B}-\mathrm{C} 6 \mathrm{~B}-\mathrm{H} 62 \mathrm{~B}$ & 109.00 \\
\hline $\mathrm{C} 3 \mathrm{~A}-\mathrm{C} 4 \mathrm{~A}-\mathrm{H} 4 \mathrm{~A}$ & 121.00 & $\mathrm{C} 5 \mathrm{~B}-\mathrm{C} 6 \mathrm{~B}-\mathrm{H} 61 \mathrm{~B}$ & 109.00 \\
\hline $\mathrm{C} 5 \mathrm{~A}-\mathrm{C} 4 \mathrm{~A}-\mathrm{H} 4 \mathrm{~A}$ & 121.00 & $\mathrm{C} 5 \mathrm{~B}-\mathrm{C} 6 \mathrm{~B}-\mathrm{H} 62 \mathrm{~B}$ & 109.00 \\
\hline $\mathrm{C} 5 \mathrm{~A}-\mathrm{C} 6 \mathrm{~A}-\mathrm{H} 6 \mathrm{~A}$ & 121.00 & $\mathrm{H} 61 \mathrm{~B}-\mathrm{C} 6 \mathrm{~B}-\mathrm{H} 62 \mathrm{~B}$ & 108.00 \\
\hline $\mathrm{C} 1 \mathrm{~A}-\mathrm{C} 6 \mathrm{~A}-\mathrm{H} 6 \mathrm{~A}$ & 121.00 & & \\
\hline $\mathrm{C} 12 \mathrm{~A}-\mathrm{O} 11 \mathrm{~A}-\mathrm{C} 1 \mathrm{~A}-\mathrm{C} 2 \mathrm{~A}$ & $-8.1(3)$ & $\mathrm{C} 1 \mathrm{~A}-\mathrm{C} 2 \mathrm{~A}-\mathrm{C} 3 \mathrm{~A}-\mathrm{C} 13 \mathrm{~A}$ & $178.48(16)$ \\
\hline $\mathrm{C} 12 \mathrm{~A}-\mathrm{O} 11 \mathrm{~A}-\mathrm{C} 1 \mathrm{~A}-\mathrm{C} 6 \mathrm{~A}$ & $172.70(18)$ & $\mathrm{C} 13 \mathrm{~A}-\mathrm{C} 3 \mathrm{~A}-\mathrm{C} 4 \mathrm{~A}-\mathrm{C} 5 \mathrm{~A}$ & $-178.43(16)$ \\
\hline $\mathrm{C} 1 \mathrm{~A}-\mathrm{O} 11 \mathrm{~A}-\mathrm{C} 12 \mathrm{~A}-\mathrm{C} 13 \mathrm{~A}$ & $-76.5(2)$ & $\mathrm{C} 2 \mathrm{~A}-\mathrm{C} 3 \mathrm{~A}-\mathrm{C} 4 \mathrm{~A}-\mathrm{C} 5 \mathrm{~A}$ & $1.5(3)$ \\
\hline
\end{tabular}




$\begin{array}{llll}\mathrm{C} 3 \mathrm{~B}-\mathrm{O} 4 \mathrm{~B}-\mathrm{C} 6 \mathrm{~B}-\mathrm{C} 5 \mathrm{~B} & 62.3(2) & \mathrm{C} 3 \mathrm{~A}-\mathrm{C} 4 \mathrm{~A}-\mathrm{C} 5 \mathrm{~A}-\mathrm{C} 15 \mathrm{~A} & -179.46(16) \\ \mathrm{C} 6 \mathrm{~B}-\mathrm{O} 4 \mathrm{~B}-\mathrm{C} 3 \mathrm{~B}-\mathrm{C} 2 \mathrm{~B} & -61.6(2) & \mathrm{C} 3 \mathrm{~A}-\mathrm{C} 4 \mathrm{~A}-\mathrm{C} 5 \mathrm{~A}-\mathrm{C} 6 \mathrm{~A} & 0.2(3) \\ \mathrm{C} 2 \mathrm{~B}-\mathrm{N} 1 \mathrm{~B}-\mathrm{C} 5 \mathrm{~B}-\mathrm{C} 6 \mathrm{~B} & 51.4(3) & \mathrm{C} 4 \mathrm{~A}-\mathrm{C} 5 \mathrm{~A}-\mathrm{C} 6 \mathrm{~A}-\mathrm{C} 1 \mathrm{~A} & -1.8(3) \\ \mathrm{C} 5 \mathrm{~B}-\mathrm{N} 1 \mathrm{~B}-\mathrm{C} 2 \mathrm{~B}-\mathrm{C} 3 \mathrm{~B} & -51.2(3) & \mathrm{C} 15 \mathrm{~A}-\mathrm{C} 5 \mathrm{~A}-\mathrm{C} 6 \mathrm{~A}-\mathrm{C} 1 \mathrm{~A} & 177.83(16) \\ \mathrm{C} 2 \mathrm{~A}-\mathrm{C} 1 \mathrm{~A}-\mathrm{C} 6 \mathrm{~A}-\mathrm{C} 5 \mathrm{~A} & 1.8(3) & \mathrm{O} 11 \mathrm{~A}-\mathrm{C} 12 \mathrm{~A}-\mathrm{C} 13 \mathrm{~A}-\mathrm{O} 13 \mathrm{~A} & 15.0(3) \\ \mathrm{O} 11 \mathrm{~A}-\mathrm{C} 1 \mathrm{~A}-\mathrm{C} 2 \mathrm{~A}-\mathrm{C} 3 \mathrm{~A} & -179.44(19) & \mathrm{O} 11 \mathrm{~A}-\mathrm{C} 12 \mathrm{~A}-\mathrm{C} 13 \mathrm{~A}-\mathrm{O} 14 \mathrm{~A} & -167.02(18) \\ \mathrm{C} 6 \mathrm{~A}-\mathrm{C} 1 \mathrm{~A}-\mathrm{C} 2 \mathrm{~A}-\mathrm{C} 3 \mathrm{~A} & -0.3(3) & \mathrm{N} 1 \mathrm{~B}-\mathrm{C} 2 \mathrm{~B}-\mathrm{C} 3 \mathrm{~B}-\mathrm{O} 4 \mathrm{~B} & 56.1(2) \\ \mathrm{O} 11 \mathrm{~A}-\mathrm{C} 1 \mathrm{~A}-\mathrm{C} 6 \mathrm{~A}-\mathrm{C} 5 \mathrm{~A} & -178.92(19) & \mathrm{N} 1 \mathrm{~B}-\mathrm{C} 5 \mathrm{~B}-\mathrm{C} 6 \mathrm{~B}-\mathrm{O} 4 \mathrm{~B} & -56.9(2) \\ \mathrm{C} 1 \mathrm{~A}-\mathrm{C} 2 \mathrm{~A}-\mathrm{C} 3 \mathrm{~A}-\mathrm{C} 4 \mathrm{~A} & -1.5(3) & & \end{array}$

Hydrogen-bond geometry $\left(\AA,{ }^{\circ}\right)$

\begin{tabular}{lllll}
\hline$D-\mathrm{H} \cdots A$ & $D-\mathrm{H}$ & $\mathrm{H} \cdots A$ & $D \cdots A$ & $D-\mathrm{H} \cdots A$ \\
\hline $\mathrm{N} 1 B-\mathrm{H} 11 B \cdots \mathrm{O} 13 A$ & $0.88(2)$ & $2.07(2)$ & $2.892(3)$ & $156(2)$ \\
$\mathrm{N} 1 B-\mathrm{H} 11 B \cdots \mathrm{O} 14 A$ & $0.88(2)$ & $2.26(2)$ & $2.988(3)$ & $141(2)$ \\
$\mathrm{N} 1 B-\mathrm{H} 12 B \cdots \mathrm{O} 14 A^{\mathrm{i}}$ & $0.88(2)$ & $1.87(2)$ & $2.737(3)$ & $170(2)$ \\
$\mathrm{C} 12 A-\mathrm{H} 12 A \cdots \mathrm{O} 13 A^{\mathrm{ii}}$ & 0.99 & 2.41 & $3.398(3)$ & 173 \\
\hline
\end{tabular}

Symmetry codes: (i) $x-1, y, z$; (ii) $x+1, y, z$.

(IV) Tetrahydro-2H-1,4-oxazin-4-ium (2,4-dichlorophenoxy)acetate

\section{Crystal data}

$\mathrm{C}_{4} \mathrm{H}_{10} \mathrm{NO}^{+} \cdot \mathrm{C}_{8} \mathrm{H}_{5} \mathrm{Cl}_{2} \mathrm{O}_{3}^{-}$

$M_{r}=308.15$

Monoclinic, $P 2_{1} / c$

Hall symbol: -P $2 \mathrm{ybc}$

$a=9.3657(5) \AA$

$b=7.1702(3) \AA$

$c=21.1340(11) \AA$

$\beta=97.981(5)^{\circ}$

$V=1405.48(12) \AA^{3}$

$Z=4$

\section{Data collection}

Oxford Diffraction Gemini-S CCD-detector diffractometer

Radiation source: Enhance (Mo) X-ray source

Graphite monochromator

Detector resolution: 16.077 pixels $\mathrm{mm}^{-1}$

$\omega$ scans

Absorption correction: multi-scan

(CrysAlis PRO; Agilent, 2014)

$T_{\min }=0.933, T_{\text {max }}=0.980$

\section{Refinement}

Refinement on $F^{2}$

Least-squares matrix: full

$R\left[F^{2}>2 \sigma\left(F^{2}\right)\right]=0.038$

$w R\left(F^{2}\right)=0.091$

$S=1.04$

2754 reflections

178 parameters

2 restraints
$F(000)=640$

$D_{\mathrm{x}}=1.456 \mathrm{Mg} \mathrm{m}^{-3}$

Mo $K \alpha$ radiation, $\lambda=0.71073 \AA$

Cell parameters from 2359 reflections

$\theta=3.6-28.4^{\circ}$

$\mu=0.47 \mathrm{~mm}^{-1}$

$T=200 \mathrm{~K}$

Plate, colourless

$0.35 \times 0.35 \times 0.12 \mathrm{~mm}$

6400 measured reflections

2754 independent reflections

2273 reflections with $I .2 \sigma(I)$

$R_{\text {int }}=0.026$

$\theta_{\text {max }}=26.0^{\circ}, \theta_{\text {min }}=3.4^{\circ}$

$h=-9 \rightarrow 11$

$k=-8 \rightarrow 8$

$l=-26 \rightarrow 20$

Primary atom site location: structure-invariant direct methods

Secondary atom site location: difference Fourier map

Hydrogen site location: inferred from neighbouring sites

$\mathrm{H}$ atoms treated by a mixture of independent and constrained refinement 
$w=1 /\left[\sigma^{2}\left(F_{\mathrm{o}}^{2}\right)+(0.0405 P)^{2}+0.3419 P\right]$

where $P=\left(F_{\mathrm{o}}^{2}+2 F_{\mathrm{c}}{ }^{2}\right) / 3$

$(\Delta / \sigma)_{\max }<0.001$

$$
\Delta \rho_{\max }=0.28 \mathrm{e} \AA^{-3}
$$

\section{Special details}

Geometry. Bond distances, angles etc. have been calculated using the rounded fractional coordinates. All su's are estimated from the variances of the (full) variance-covariance matrix. The cell esds are taken into account in the estimation of distances, angles and torsion angles

Refinement. Refinement of $\mathrm{F}^{2}$ against ALL reflections. The weighted R-factor $\mathrm{wR}$ and goodness of fit $\mathrm{S}$ are based on $\mathrm{F}^{2}$, conventional R-factors $\mathrm{R}$ are based on $\mathrm{F}$, with $\mathrm{F}$ set to zero for negative $\mathrm{F}^{2}$. The threshold expression of $\mathrm{F}^{2}>2$ sigma $\left(\mathrm{F}^{2}\right)$ is used only for calculating R-factors(gt) etc. and is not relevant to the choice of reflections for refinement. R-factors based on $\mathrm{F}^{2}$ are statistically about twice as large as those based on F, and R- factors based on ALL data will be even larger.

Fractional atomic coordinates and isotropic or equivalent isotropic displacement parameters $\left(\AA^{2}\right)$

\begin{tabular}{|c|c|c|c|c|}
\hline & $x$ & $y$ & $z$ & $U_{\text {iso }} * / U_{\text {eq }}$ \\
\hline $\mathrm{Cl} 2 \mathrm{~A}$ & $0.23659(5)$ & $0.74483(6)$ & $0.41042(2)$ & $0.0354(2)$ \\
\hline C14A & $0.15479(6)$ & $0.32605(9)$ & 0.19759 (2) & $0.0500(2)$ \\
\hline O11A & $0.13251(13)$ & $0.42531(17)$ & $0.47171(6)$ & $0.0276(4)$ \\
\hline O13A & $0.34279(13)$ & $0.1659(2)$ & $0.50395(7)$ & $0.0390(5)$ \\
\hline O14A & $0.19747(12)$ & $-0.01154(18)$ & $0.55307(6)$ & $0.0313(4)$ \\
\hline $\mathrm{C} 1 \mathrm{~A}$ & $0.13210(17)$ & $0.3923(3)$ & $0.40846(8)$ & $0.0236(5)$ \\
\hline $\mathrm{C} 2 \mathrm{~A}$ & $0.18091(18)$ & 0.5368 & $0.37274(9)$ & $0.0255(5)$ \\
\hline $\mathrm{C} 3 \mathrm{~A}$ & 0.18796 (19) & 0.5185 & $0.30830(9)$ & $0.0305(6)$ \\
\hline $\mathrm{C} 4 \mathrm{~A}$ & 0.14433 (19) & 0.3520 & $0.27899(9)$ & $0.0319(6)$ \\
\hline $\mathrm{C} 5 \mathrm{~A}$ & $0.0933(2)$ & 0.2087 & $0.31247(10)$ & $0.0348(6)$ \\
\hline C6A & 0.08662 (19) & 0.2287 & $0.37705(9)$ & $0.0306(6)$ \\
\hline $\mathrm{C} 12 \mathrm{~A}$ & 0.10285 (19) & 0.2707 & $0.51091(9)$ & $0.0270(6)$ \\
\hline C13A & $0.22590(18)$ & $0.1309(2)$ & $0.52275(8)$ & $0.0226(5)$ \\
\hline O4B & 0.56703 (19) & $0.3986(3)$ & $0.31170(7)$ & $0.0591(6)$ \\
\hline N1B & $0.55499(17)$ & $0.2047(2)$ & $0.42729(8)$ & $0.0293(5)$ \\
\hline $\mathrm{C} 2 \mathrm{~B}$ & $0.5720(2)$ & $0.4092(3)$ & $0.42590(10)$ & $0.0353(7)$ \\
\hline C3B & $0.6444(3)$ & $0.4633(3)$ & $0.37002(11)$ & $0.0509(8)$ \\
\hline C5B & $0.5609(3)$ & $0.2016(4)$ & $0.31253(11)$ & $0.0541(9)$ \\
\hline C6B & $0.4830(2)$ & $0.1326(3)$ & $0.36568(11)$ & $0.0412(7)$ \\
\hline $\mathrm{H} 3 \mathrm{~A}$ & 0.22200 & 0.61780 & 0.28470 & $0.0370^{*}$ \\
\hline H5A & 0.06260 & 0.09580 & 0.29130 & $0.0420 *$ \\
\hline H6A & 0.05060 & 0.12950 & 0.40010 & $0.0370^{*}$ \\
\hline $\mathrm{H} 12 \mathrm{~A}$ & 0.01550 & 0.20560 & 0.49020 & $0.0320 *$ \\
\hline H13A & 0.08180 & 0.31880 & 0.55260 & $0.0320^{*}$ \\
\hline H11B & $0.6420(17)$ & $0.149(3)$ & $0.4381(9)$ & $0.0350 *$ \\
\hline $\mathrm{H} 12 \mathrm{~B}$ & $0.507(2)$ & $0.180(3)$ & $0.4587(8)$ & $0.0350^{*}$ \\
\hline $\mathrm{H} 21 \mathrm{~B}$ & 0.63050 & 0.45170 & 0.46590 & $0.0420^{*}$ \\
\hline $\mathrm{H} 22 \mathrm{~B}$ & 0.47630 & 0.46970 & 0.42270 & $0.0420^{*}$ \\
\hline H31B & 0.65250 & 0.60090 & 0.36850 & $0.0610^{*}$ \\
\hline H32B & 0.74300 & 0.41080 & 0.37530 & $0.0610^{*}$ \\
\hline H51B & 0.66010 & 0.15050 & 0.31820 & $0.0650^{*}$ \\
\hline H52B & 0.51060 & 0.15630 & 0.27110 & $0.0650^{*}$ \\
\hline H61B & 0.38150 & 0.17540 & 0.35850 & $0.0490^{*}$ \\
\hline
\end{tabular}


Atomic displacement parameters $\left(\AA^{2}\right)$

\begin{tabular}{lllllll}
\hline & $U^{11}$ & $U^{22}$ & $U^{33}$ & $U^{12}$ & $U^{13}$ & $U^{23}$ \\
\hline C12A & $0.0474(3)$ & $0.0221(3)$ & $0.0376(3)$ & $-0.0043(2)$ & $0.0088(2)$ & $0.0006(2)$ \\
C14A & $0.0576(4)$ & $0.0620(4)$ & $0.0312(3)$ & $0.0069(3)$ & $0.0087(2)$ & $-0.0094(3)$ \\
O11A & $0.0339(7)$ & $0.0222(7)$ & $0.0274(7)$ & $0.0037(6)$ & $0.0072(5)$ & $0.0055(6)$ \\
O13A & $0.0247(7)$ & $0.0429(9)$ & $0.0524(9)$ & $0.0066(6)$ & $0.0162(6)$ & $0.0154(7)$ \\
O14A & $0.0291(7)$ & $0.0282(7)$ & $0.0368(8)$ & $0.0031(6)$ & $0.0056(6)$ & $0.0117(6)$ \\
C1A & $0.0202(8)$ & $0.0236(9)$ & $0.0265(10)$ & $0.0059(7)$ & $0.0019(7)$ & $0.0052(8)$ \\
C2A & $0.0228(9)$ & $0.0222(9)$ & $0.0315(10)$ & $0.0032(8)$ & $0.0036(7)$ & $0.0015(8)$ \\
C3A & $0.0290(10)$ & $0.0325(11)$ & $0.0307(11)$ & $0.0034(9)$ & $0.0063(8)$ & $0.0071(9)$ \\
C4A & $0.0295(10)$ & $0.0386(12)$ & $0.0268(10)$ & $0.0064(9)$ & $0.0010(8)$ & $-0.0019(9)$ \\
C5A & $0.0328(10)$ & $0.0302(11)$ & $0.0389(12)$ & $-0.0021(9)$ & $-0.0040(9)$ & $-0.0053(9)$ \\
C6A & $0.0283(10)$ & $0.0260(10)$ & $0.0363(11)$ & $-0.0026(8)$ & $0.0003(8)$ & $0.0041(9)$ \\
C12A & $0.0259(9)$ & $0.0278(10)$ & $0.0288(10)$ & $0.0031(8)$ & $0.0090(7)$ & $0.0080(8)$ \\
C13A & $0.0236(9)$ & $0.0225(9)$ & $0.0214(9)$ & $0.0011(8)$ & $0.0025(7)$ & $-0.0005(8)$ \\
O4B & $0.0774(12)$ & $0.0641(12)$ & $0.0344(9)$ & $-0.0058(10)$ & $0.0026(8)$ & $0.0203(9)$ \\
N1B & $0.0245(8)$ & $0.0311(9)$ & $0.0338(9)$ & $0.0047(7)$ & $0.0089(7)$ & $0.0114(8)$ \\
C2B & $0.0385(11)$ & $0.0292(11)$ & $0.0367(12)$ & $0.0044(9)$ & $0.0001(9)$ & $0.0014(9)$ \\
C3B & $0.0611(15)$ & $0.0443(14)$ & $0.0466(14)$ & $-0.0155(12)$ & $0.0046(11)$ & $0.0164(12)$ \\
C5B & $0.0596(15)$ & $0.0678(18)$ & $0.0337(13)$ & $0.0014(14)$ & $0.0025(11)$ & $-0.0126(13)$ \\
C6B & $0.0362(11)$ & $0.0337(12)$ & $0.0524(14)$ & $-0.0035(10)$ & $0.0012(10)$ & $-0.0015(10)$ \\
& & & & & &
\end{tabular}

Geometric parameters $\left(\AA,{ }^{\circ}\right)$

\begin{tabular}{llll}
\hline $\mathrm{C} 12 \mathrm{~A}-\mathrm{C} 2 \mathrm{~A}$ & $1.737(2)$ & $\mathrm{C} 5 \mathrm{~A}-\mathrm{C} 6 \mathrm{~A}$ & $1.382(3)$ \\
$\mathrm{C} 14 \mathrm{~A}-\mathrm{C} 4 \mathrm{~A}$ & $1.7466(19)$ & $\mathrm{C} 12 \mathrm{~A}-\mathrm{C} 13 \mathrm{~A}$ & $1.522(3)$ \\
$\mathrm{O} 11 \mathrm{~A}-\mathrm{C} 1 \mathrm{~A}$ & $1.357(2)$ & $\mathrm{C} 3 \mathrm{~A}-\mathrm{H} 3 \mathrm{~A}$ & 0.9500 \\
$\mathrm{O} 11 \mathrm{~A}-\mathrm{C} 12 \mathrm{~A}$ & $1.434(2)$ & $\mathrm{C} 5 \mathrm{~A}-\mathrm{H} 5 \mathrm{~A}$ & 0.9500 \\
$\mathrm{O} 13 \mathrm{~A}-\mathrm{C} 13 \mathrm{~A}$ & $1.241(2)$ & $\mathrm{C} 6 \mathrm{~A}-\mathrm{H} 6 \mathrm{~A}$ & 0.9500 \\
$\mathrm{O} 14 \mathrm{~A}-\mathrm{C} 13 \mathrm{~A}$ & $1.254(2)$ & $\mathrm{C} 12 \mathrm{~A}-\mathrm{H} 12 \mathrm{~A}$ & 0.9900 \\
$\mathrm{O} 4 \mathrm{~B}-\mathrm{C} 5 \mathrm{~B}$ & $1.414(4)$ & $\mathrm{C} 12 \mathrm{~A}-\mathrm{H} 13 \mathrm{~A}$ & 0.9900 \\
$\mathrm{O} 4 \mathrm{~B}-\mathrm{C} 3 \mathrm{~B}$ & $1.418(3)$ & $\mathrm{C} 2 \mathrm{~B}-\mathrm{C} 3 \mathrm{~B}$ & $1.492(3)$ \\
$\mathrm{N} 1 \mathrm{~B}-\mathrm{C} 2 \mathrm{~B}$ & $1.476(3)$ & $\mathrm{C} 5 \mathrm{~B}-\mathrm{C} 6 \mathrm{~B}$ & $1.506(3)$ \\
$\mathrm{N} 1 \mathrm{~B}-\mathrm{C} 6 \mathrm{~B}$ & $1.474(3)$ & $\mathrm{C} 2 \mathrm{~B}-\mathrm{H} 21 \mathrm{~B}$ & 0.9900 \\
$\mathrm{~N} 1 \mathrm{~B}-\mathrm{H} 12 \mathrm{~B}$ & $0.870(18)$ & $\mathrm{C} 2 \mathrm{~B}-\mathrm{H} 22 \mathrm{~B}$ & 0.9900 \\
$\mathrm{~N} 1 \mathrm{~B}-\mathrm{H} 11 \mathrm{~B}$ & $0.908(18)$ & $\mathrm{C} 3 \mathrm{~B}-\mathrm{H} 31 \mathrm{~B}$ & 0.9900 \\
$\mathrm{C} 1 \mathrm{~A}-\mathrm{C} 2 \mathrm{~A}$ & $1.396(3)$ & $\mathrm{C} 3 \mathrm{~B}-\mathrm{H} 32 \mathrm{~B}$ & 0.9900 \\
$\mathrm{C} 1 \mathrm{~A}-\mathrm{C} 6 \mathrm{~A}$ & $1.386(3)$ & $\mathrm{C} 5 \mathrm{~B}-\mathrm{H} 51 \mathrm{~B}$ & 0.9900 \\
$\mathrm{C} 2 \mathrm{~A}-\mathrm{C} 3 \mathrm{~A}$ & $1.379(3)$ & $\mathrm{C} 5 \mathrm{~B}-\mathrm{H} 52 \mathrm{~B}$ & 0.9900 \\
$\mathrm{C} 3 \mathrm{~A}-\mathrm{C} 4 \mathrm{~A}$ & $1.380(3)$ & $\mathrm{C} 6 \mathrm{~B}-\mathrm{H} 61 \mathrm{~B}$ & 0.9900 \\
$\mathrm{C} 4 \mathrm{~A}-\mathrm{C} 5 \mathrm{~A}$ & $1.371(3)$ & $\mathrm{C} 6 \mathrm{~B}-\mathrm{H} 62 \mathrm{~B}$ & 0.9900 \\
& & & \\
$\mathrm{C} 1 \mathrm{~A}-\mathrm{O} 11 \mathrm{~A}-\mathrm{C} 12 \mathrm{~A}$ & $117.39(15)$ & $\mathrm{C} 13 \mathrm{~A}-\mathrm{C} 12 \mathrm{~A}-\mathrm{H} 12 \mathrm{~A}$ & 109.00 \\
$\mathrm{C} 3 \mathrm{~B}-\mathrm{O} 4 \mathrm{~B}-\mathrm{C} 5 \mathrm{~B}$ & $109.44(17)$ & O11A-C12A-H12A & 109.00 \\
$\mathrm{C} 2 \mathrm{~B}-\mathrm{N} 1 \mathrm{~B}-\mathrm{C} 6 \mathrm{~B}$ & $111.61(16)$ & $\mathrm{C} 13 \mathrm{~A}-\mathrm{C} 12 \mathrm{~A}-\mathrm{H} 13 \mathrm{~A}$ & 109.00
\end{tabular}




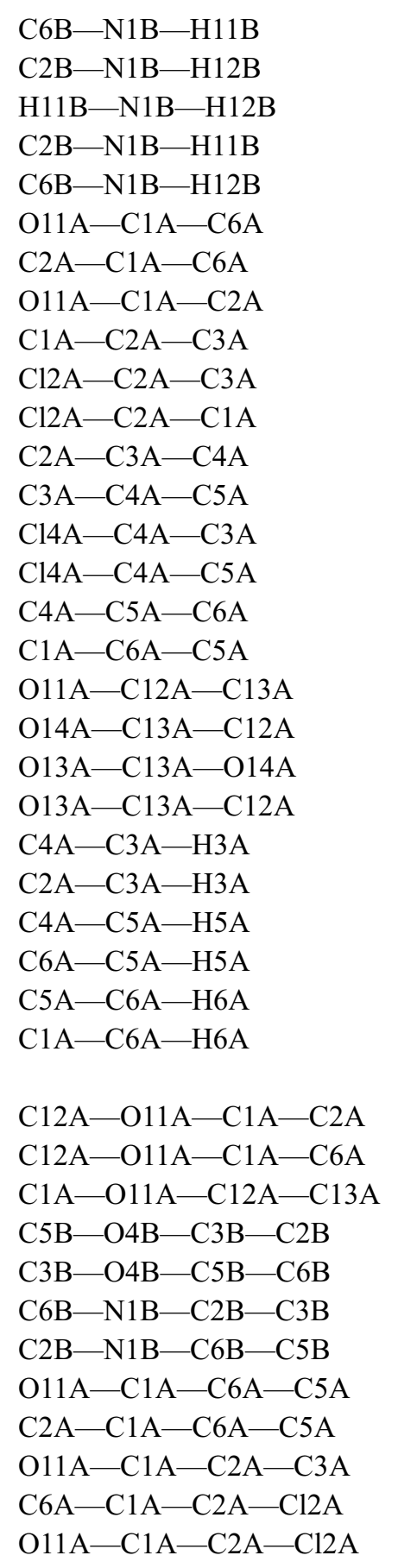

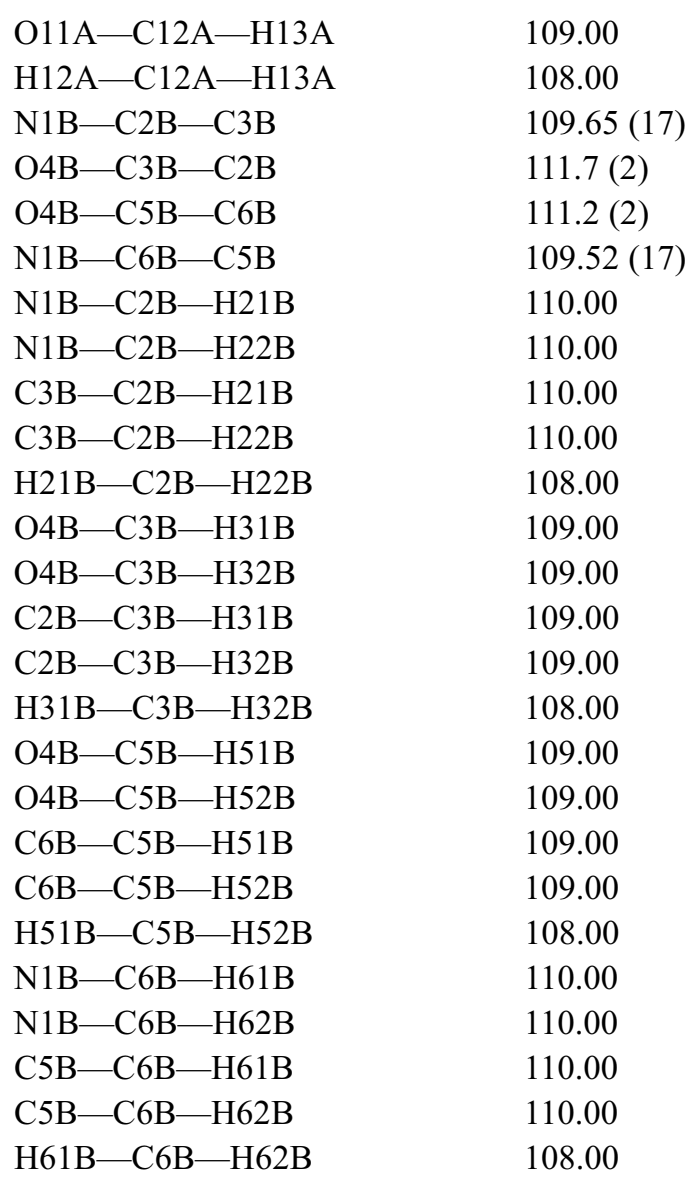

109.00

108.00

109.65 (17)

111.7 (2)

$111.2(2)$

$109.52(17)$

110.00

110.00

110.00

110.00

108.00

109.00

109.00

109.00

109.00

108.00

109.00

109.00

109.00

109.00

108.00

110.00

110.00

110.00

110.00

108.00

$-1.6(3)$

$179.56(14)$

0.4 (3)

$-179.24(14)$

0.9 (3)

$179.27(15)$

$-0.9(3)$

$-0.5(3)$

$6.5(2)$

$-175.12(14)$

$57.1(2)$

$-57.3(2)$

Hydrogen-bond geometry $\left(A,{ }^{\circ}\right)$

\begin{tabular}{lllll}
\hline$D-\mathrm{H} \cdots A$ & $D-\mathrm{H}$ & $\mathrm{H} \cdots A$ & $D \cdots A$ & $D-\mathrm{H} \cdots A$ \\
\hline $\mathrm{N} 1 B-\mathrm{H} 11 B \cdots \mathrm{O} 13 A^{\mathrm{i}}$ & $0.91(2)$ & $2.56(2)$ & $3.115(2)$ & $120(1)$ \\
$\mathrm{N} 1 B-\mathrm{H} 11 B \cdots \mathrm{O} 14 A^{\mathrm{i}}$ & $0.91(2)$ & $1.79(2)$ & $2.683(2)$ & $169(2)$ \\
$\mathrm{N} 1 B-\mathrm{H} 12 B \cdots \mathrm{O} 13 A$ & $0.87(2)$ & $1.92(2)$ & $2.747(2)$ & $158(2)$ \\
$\mathrm{C} 12 A-\mathrm{H} 12 A \cdots \mathrm{O} 14 A^{\mathrm{ii}}$ & 0.99 & 2.50 & $3.484(2)$ & 173
\end{tabular}


supporting information

\begin{tabular}{llllr}
$\mathrm{C} 2 B-\mathrm{H} 21 B \cdots \mathrm{O} 11 A^{\mathrm{iii}}$ & 0.99 & 2.57 & $3.477(2)$ & 151 \\
$\mathrm{C} 5 B-\mathrm{H} 52 B \cdots \mathrm{O} 4 B^{\mathrm{iv}}$ & 0.99 & 2.58 & $3.489(3)$ & 153 \\
\hline
\end{tabular}

Symmetry codes: (i) $-x+1,-y,-z+1$; (ii) $-x,-y,-z+1$; (iii) $-x+1,-y+1,-z+1$; (iv) $-x+1, y-1 / 2,-z+1 / 2$. 